\title{
APOE genotype dependent molecular abnormalities in the cerebrovasculature of Alzheimer's disease and age-matched non-demented brains
}

Joseph O. Ojo ${ }^{1,2,3^{*}} \mathbb{D}$, Jon M. Reed ${ }^{1,4}$, Gogce Crynen ${ }^{1}$, Prashanthi Vallabhaneni ${ }^{1}$, James Evans ${ }^{1}$, Benjamin Shackleton ${ }^{1,3}$, Maximillian Eisenbaum ${ }^{1,3}$, Charis Ringland ${ }^{1,3}$, Anastasia Edsell ${ }^{1}$, Michael Mullan ${ }^{1,3}$, Fiona Crawford ${ }^{1,2,3}$ and Corbin Bachmeier ${ }^{1,3,5}$

\begin{abstract}
Cerebrovascular dysfunction is a hallmark feature of Alzheimer's disease (AD). One of the greatest risk factors for AD is the apolipoprotein E4 (E4) allele. The APOE4 genotype has been shown to negatively impact vascular amyloid clearance, however, its direct influence on the molecular integrity of the cerebrovasculature compared to other APOE variants (APOE2 and APOE3) has been largely unexplored. To address this, we employed a 10-plex tandem isobaric mass tag approach in combination with an ultra-high pressure liquid chromatography MS/MS (Q-Exactive) method, to interrogate unbiased proteomic changes in cerebrovessels from $A D$ and healthy control brains with different APOE genotypes. We first interrogated changes between healthy control cases to identify underlying genotype specific effects in cerebrovessels. EIF2 signaling, regulation of elF4 and 70S6K signaling and mTOR signaling were the top significantly altered pathways in E4/E4 compared to E3/E3 cases. Oxidative phosphorylation, EIF2 signaling and mitochondrial dysfunction were the top significant pathways in E2E2 vs E3/E3cases. We also identified AD-dependent changes and their interactions with APOE genotype and found the highest number of significant proteins from this interaction was observed in the E3/E4 (192) and E4/E4 (189) cases. As above, EIF2, mTOR signaling and elF4 and 70S6K signaling were the top three significantly altered pathways in E4 allele carriers (i.e. E3/E4 and E4/E4 genotypes). Of all the cerebrovascular cell-type specific markers identified in our proteomic analyses, endothelial cell, astrocyte, and smooth muscle cell specific protein markers were significantly altered in E3/E4 cases, while endothelial cells and astrocyte specific protein markers were altered in E4/E4 cases. These proteomic changes provide novel insights into the longstanding link between APOE4 and cerebrovascular dysfunction, implicating a role for impaired autophagy, ER stress, and mitochondrial bioenergetics. These APOE4 dependent changes we identified could provide novel cerebrovascular targets for developing disease modifying strategies to mitigate the effects of APOE4 genotype on AD pathogenesis.
\end{abstract}

Keywords: Cerebrovasculature, APOE, Aging, Alzheimer's disease, Mural cells, Endothelial cells, Proteomics, Mass spectrometry

*Correspondence: jojo@roskampinstitute.org

1 Department of Experimental Neuropathology, Roskamp Institute, Sarasota, FL 34243, USA

Full list of author information is available at the end of the article

\section{Introduction}

Alzheimer's disease (AD) is the most predominant type of dementia, which to date remains untreatable. One of the most common preclinical features of $\mathrm{AD}$ is vascular original author(s) and the source, provide a link to the Creative Commons licence, and indicate if changes were made. The images or other third party material in this article are included in the article's Creative Commons licence, unless indicated otherwise in a credit line to the material. If material is not included in the article's Creative Commons licence and your intended use is not permitted by statutory regulation or exceeds the permitted use, you will need to obtain permission directly from the copyright holder. To view a copy of this licence, visit http://creativecommons.org/licenses/by/4.0/. The Creative Commons Public Domain Dedication waiver (http://creativeco mmons.org/publicdomain/zero/1.0/) applies to the data made available in this article, unless otherwise stated in a credit line to the data. 
dysfunction, evident by deficits in cortical blood flow and metabolic activity, many years before the onset of neurological symptoms [1-5]. At autopsy, vascular lesions are routinely reported in early prodromal and later stages of AD. This is typified by cerebral amyloid angiopathy (CAA) [6], lesions of cerebral small vessel disease (CSVD) such as lacunar infarcts and microhemorrhages [7-9], cerebral atherosclerosis [10-12], degenerating small blood vessels, capillaries and mural cells [13-17], weakened blood-brain barrier (BBB) [18], and buildup of phagolysosomes-lipofuscin deposits and dysmorphic mitochondria in cerebrovascular cells $[3,16,19]$. Some of these vascular lesions can act as protagonists negatively modifying the clinical presentation of AD [7-9, 20-22].

It remains unknown whether these vascular changes in $\mathrm{AD}$ are a prelude to, or a direct consequence of $\mathrm{A} \beta$ toxicity [23]. Early vascular dysfunction (pre-amyloidosis) can contribute to an increase in $A \beta$ accumulation in leptomeninges and along cerebral blood vessels as perivascular drainage along these sites is a preferred route of amyloid clearance from the brain [23-25]. Deposition of A $\beta$ on vessel walls can also induce cerebrovascular changes that further impairs vascular hemodynamics [26, 27], increasing cerebral blood pressure and reducing cerebral perfusion $[28,29]$. The specific molecular triggers and factors driving these degenerative cerebrovascular phenotypes and the timing of these events in the sequelae of $\mathrm{AD}$ remain elusive.

One of the strongest risk factors for $\mathrm{AD}$ is the $\varepsilon 4$ allele of the apolipoprotein E (APOE4) genotype [30-32]. The APOE protein is a multifunctional protein which contains three distinct functional domains, an $\mathrm{N}$-terminal receptor binding domain, a random coil region, and the C-terminal lipid-binding domain. The different isoforms of APOE (E2, E3, E4) differ by a single amino acid change which impacts their lipid binding capabilities. In the brain, APOE is produced and secreted primarily by astrocytes and microglia and it is subsequently lipidated to form nascent high-density lipoprotein (HDL)-like particles. This lipidated form of APOE plays a vital role in cholesterol transport, lipid metabolism [33], and $A \beta$ metabolism and clearance [30, 34].

Some of the early clues linking APOE with vascular pathobiology involved early antibody trials showing a link between the APOE4 allele and recurrent hemorrhages in cerebral amyloid angiopathy (CAA) patients [35-37]. Since then postmortem studies have revealed a correlation between APOE4 and vascular lesions such as CAA [38, 39], CSVD [40-42], atherosclerosis [43, 44] and deficits in cerebral blood flow [45]. More recent work has confirmed that the APOE4 allele accelerates BBB breakdown in elderly unimpaired individuals and, to a greater extent, in demented patients [42, 46-49].
Preclinical models expressing human APOE isoforms, have also shown that the E4 allele drives early pericyte degeneration and $\mathrm{BBB}$ abnormalities prior to neuronal dysfunction [42]. Thus it appears that APOE may significantly contribute to the degenerative cerebrovascular phenotypes in AD. Yet the specific details of how APOE isoform variants influence the molecular integrity of the cerebrovasculature in normal and pathogenic (AD) settings remains elusive.

To address this, we will use our state-of-the-art unbiased proteomic (mass spectrometry) based platform to conduct a detailed characterization and assessment of molecular changes in protein expression levels, molecular pathways and biofunctions significantly altered in the cerebrovessels of $\mathrm{AD}$ patients compared to healthy control cases from different APOE genotype backgrounds (E2/E2, E2/E3, E3/E3, E3/E4 and E4/E4). Unbiased proteomic analysis is an extremely powerful tool which can provide a very expansive interrogation of the molecular response in neurodegenerative diseases [50-53] and can lead to the identification of pathogenic mechanisms and novel molecular targets [54-56] for therapeutic exploration. Herein, we have utilized a novel protein extraction protocol, separating isolated cerebrovessels into cytosolic, membrane and nuclear fractions to increase the depth of the protein mining process, and coupled this with a 10-plex tandem isobaric mass tag (TMT) approach for interrogation with an ultra-high pressure liquid chromatography MS/MS (Q-Exactive) method [57]. In this study, we have detailed the unique molecular profiles and pathogenic mechanisms driven by APOE variant genotypes in cerebrovessels of the inferior frontal gyrus of $\mathrm{AD}$ and control patients.

\section{Methods}

\section{Human brain tissue and patient demographics}

Human brain tissue from the inferior frontal gyrus were provided mainly from Dr. Thomas Beach, Director of the Brain and Body Donation Program at Sun Health Research Institute (Sun City, AZ) in accordance with the institutional bioethics guidelines. Additional samples were requested from the NIH BrainBank repository (University of Maryland and Ican school of medicine, Mount Sinai, NY). Autopsies were conducted within 4-5 h after death from non-demented control subjects with no history of AD diagnosis, and AD subjects. Neuropathological post-mortem diagnosis of $\mathrm{AD}$ was determined using the Consortium to Establish a Registry for Alzheimer's Disease (CERAD) diagnostic criteria and the consensus recommendation by the National Institute for Aging/Reagan Institute Working Group. Braak staging was used to characterize the neurofibrillary tangle (NFT) distribution. The severity of CAA was performed 
according to Vonsattel et al. [58], and the stage of topographical expansion of CAA was assessed as previously described by Thal et al. [27] based on a 4 point numerical conversion per region. Global scores for amyloid, tangle and CAA burden from the microscopic lesion densities were calculated based on the sums of the scores from all regions interrogated. A summary of patient demographics, clinical information and APOE genotype background of brain donors used in the study is provided in Tables 1 , 2 .

\section{Isolation of enriched cerebrovessel fractions}

Enriched cerebrovessels were isolated from the inferior frontal gyrus based on a previous protocol described by our group [59, 60]. Briefly, frozen $500 \mathrm{mg}$ blocks of brain tissue from the inferior frontal gyrus was homogenized in ice-cold Hanks Buffered salt solution (HBBS) using a glass dounce homogenizer, and 6-8 passes of a Teflon pestle tissue grinder. A solution containing $40 \%$ dextran was added to the brain homogenate at an equal volume, to generate a final concentration of $20 \%$ dextran, which was subsequently centrifuged at $6000 \mathrm{~g}$ for $15 \mathrm{~min}$ at $4{ }^{\circ} \mathrm{C}$. Three visible layers were produced after centrifugation; the top 'paraenchyma fraction' layer consisted of a compact mass, the bottom 'cerebrovessel fraction' layer consisted of a tissue pellet (Fig. 1A), and this was separated by a middle layer of translucent dextran interface consisting of the non-cell associated soluble fractions. For subsequent analyses we used the bottom layer consisting of the whole cerebrovascular fraction, containing vessels with a variety of sizes (microvessels, arterioles, etc.). This fraction was highly enriched in endothelial cells, pericytes, smooth muscle cells, and to a lesser extent astrocytes.

Subcellular protein extraction from vascular homogenates To each cerebrovascular pellet, we added 250 ul of ice cold phosphate buffered saline (PBS), followed by

Table 1 List of control and Alzheimer's disease cases, their demographics, APOE genotype, and randomization of samples for Tandem Mass Tag isobaric 10-plex multiplexing

\begin{tabular}{|c|c|c|c|c|c|c|}
\hline DX & APOE genotype & Mean age [years] & Male & Female & $\mathrm{N}$ per group & TMT 10 Plex labels \\
\hline Control & $\mathrm{E} 2 / \mathrm{E} 2$ & $71.5 \pm 8.58$ & $33.3 \%(2 / 6)$ & $66.7 \%(4 / 6)$ & 6 & $\mathrm{TMT}-126$ \\
\hline Control & E2/E3 & $80 \pm 5.85$ & $50 \%(3 / 6)$ & $50 \%(3 / 6)$ & 6 & TMT -127N \\
\hline Control & E3/E3 & $83 \pm 3.04$ & $100 \%(6 / 6)$ & $0 \%(0 / 6)$ & 6 & TMT -127C* (denominator) \\
\hline Control & $\mathrm{E} 3 / \mathrm{E} 4$ & $84.17 \pm 3.64$ & $60 \%(3 / 5)$ & $40 \%(2 / 5)$ & 5 & TMT -128N \\
\hline Control & E4/E4 & $45.83 \pm 4.39$ & $66.7 \%(4 / 6)$ & $33.3 \%(2 / 6)$ & 6 & TMT -128C \\
\hline$A D$ & E2/E2 & $90 \pm 17.0^{*}$ & $0 \%(0 / 3)$ & $100 \%(3 / 3)$ & 3 & TMT -129N \\
\hline$A D$ & E2/E3 & $89 \pm 4.14$ & $66.7 \%(4 / 6)$ & $33.3 \%(2 / 6)$ & 6 & TMT -129C \\
\hline$A D$ & E3/E3 & $87 \pm 3.27$ & $16.67 \%(1 / 6)$ & $83 \%(5 / 6)$ & 6 & TMT -130N \\
\hline$A D$ & $\mathrm{E} 3 / \mathrm{E} 4$ & $78.5 \pm 3.60$ & $50 \%(3 / 6)$ & $50 \%(3 / 6)$ & 6 & TMT -130C \\
\hline$A D$ & E4/E4 & $84.8 \pm 4.55$ & $40 \%(2 / 5)$ & $60 \%(3 / 5)$ & 5 & TMT -131 \\
\hline Total Control & All Genotype & $72.90 \pm 3.49$ & $37.9 \%(18 / 29)$ & $62.1 \%(11 / 29)$ & 29 & $-126,-127 N,-127 C,-128 N,-128 C$ \\
\hline Total AD & All Genotype & $85.24 \pm 2.10$ & $38.5 \%(10 / 26)$ & $61.5 \%(16 / 26)$ & 26 & $-129 N,-129 C_{,}-130 N,-130 C_{,}-131$ \\
\hline
\end{tabular}

Table 2 Shows the clinical background of patients focusing on brain weight and scores for the mini-mental state examination (MMSE), Amyloid plaque, Tangle, Braak staging, White matter and CAA

\begin{tabular}{|c|c|c|c|c|c|c|c|c|}
\hline DX & APOE genotype & Brain weight & MMSE score & $\begin{array}{l}\text { Amyloid plaque } \\
\text { score }\end{array}$ & Tangle score & Braak score & $\begin{array}{l}\text { White matter } \\
\text { score }\end{array}$ & CAA score \\
\hline Control & E2/E2 & $1207.33 \pm 45.8$ & 29 (from 1 case) & $3.63 \pm 2.4$ & $3.25 \pm 1.8$ & $2.75 \pm 0.3$ & $2.5 \pm 1.5$ & $4 \pm 4(50 \%)$ \\
\hline Control & E2/E3. & $1286 \pm 48.8$ & $28.6 \pm 0.94$ & $1.66 \pm 1.1$ & $3.54 \pm 0.6$ & $2.17 \pm 0.4$ & $2.5 \pm 0.7$ & $0(0 \%)$ \\
\hline Control & E3/E3 & $1222 \pm 34.7$ & $28 \pm 0.96$ & $1.33 \pm 1.3$ & $2.67 \pm 0.79$ & $2.17 \pm 0.4$ & $1.67 \pm 0.6$ & $2.83 \pm 1.9(40 \%)$ \\
\hline Control & E3/E4 & $1193.75 \pm 82.4$ & $28.25 \pm 0.3$ & $3.75 \pm 2.4$ & $4.21 \pm 0.9$ & $3 \pm 0.3$ & $3.25 \pm 1.9$ & $0.4 \pm 0.4(20 \%)$ \\
\hline Control & E4/E4 & $1310 \pm 40$ & $\mathrm{~N} / \mathrm{A}$ & $\mathrm{N} / \mathrm{A}$ & $\mathrm{N} / \mathrm{A}$ & $\mathrm{N} / \mathrm{A}$ & $\mathrm{N} / \mathrm{A}$ & $\mathrm{N} / \mathrm{A}$ \\
\hline$A D$ & E2/E2 & $\mathrm{N} / \mathrm{A}$ & $\mathrm{N} / \mathrm{A}$ & $7.83 \pm 2.4$ & 15 (from 1 case) & $5.67 \pm 0.3$ & 6 (from 1 case) & 12 (from 1 case) \\
\hline$A D$ & E2/E3 & $1104.83 \pm 80.9$ & $18.3 \pm 3.0$ & $9.3 \pm 1.7$ & $8 \pm 1.1$ & $4.16 \pm 0.2$ & $3.3 \pm 0.8$ & $4.17 \pm 1.7(60 \%)$ \\
\hline$A D$ & E3/E3 & $1060 \pm 37.9$ & $6.67 \pm 5.7$ & $13.04 \pm 0.97$ & $10.4 \pm 2.12$ & $4.67 \pm 0.61$ & $5.83 \pm 1.9$ & $3.4 \pm 1.3(66.6 \%)$ \\
\hline$A D$ & E3/E4 & $1036.8 \pm 49.3$ & $18 \pm 3.2$ & $13 \pm 0.6$ & $13 \pm 0.9$ & $5.167 \pm 0.3$ & $2.8 \pm 1.3$ & $4.6 \pm 0.9(100 \%)$ \\
\hline$A D$ & E4/E4 & $959 \pm 18.1$ & $11 \pm 2$ & $14.25 \pm 0.31$ & $14.5 \pm 0.32$ & $6 \pm 0$ & $6.2 \pm 0.9$ & $8.33 \pm 1.4(100 \%)$ \\
\hline
\end{tabular}


homogenization using a probe sonicator and subsequent centrifugation at $20,000 \mathrm{~g}$ for $5 \mathrm{~min}$ at $4{ }^{\circ} \mathrm{C}$. Supernatants were collected in tubes to obtain the PBS-fraction. Pelleted samples were re-suspended in $250 \mathrm{ul}$ of ice cold PBS containing $1 \mathrm{M}$ sodium chloride, further sonicated and centrifuged at 20,000 g for $5 \mathrm{~min}$ at $4{ }^{\circ} \mathrm{C}$. Supernatant was collected in a different tube and labeled as PBS-high salt fraction. The precipitant was resuspended in $250 \mathrm{ul}$ of ice cold $20 \mathrm{mM}$ Triethylamonium bicarbonate (TEAB) and $2 \%$ lithium dodecylsulphate anionic detergent, sonicated, and underwent centrifugation at 20,000 $\mathrm{g}$ for $5 \mathrm{~min}$ at $4{ }^{\circ} \mathrm{C}$. Final supernatant was transferred to a new Eppendorf tube and labeled as the membrane protein pellet fraction. Twelve and a half microliters of $21 \times$ proteinase inhibitor cocktail was added to $250 \mathrm{ul}$ of all the three fractions. To enhance the proteomic mining process, we used all three fractions-PBS, PBS-high salt and membrane fractions (i.e. cytosolic, nuclear and membrane proteins) - for the entire study.

\section{Trypsin digestion}

BCA analyses was used to determine protein concentration prior to trypsin digestion. For the PBS fraction, $30 \mathrm{ug}$ protein was added to $3 \times$ volume of acetone, and left to incubate at $-20{ }^{\circ} \mathrm{C}$ for $1 \mathrm{~h}$. Following centrifugation at $14,000 \mathrm{~g}$ for $1.5 \mathrm{~min}$ at room temperature, pelleted samples were brought up in $20 \mathrm{ul}$ modified reduction alkylation buffer (MRAB) consisting of $20 \mathrm{mM} \mathrm{TEAB}$ at $\mathrm{pH} 8$, $1 \% \mathrm{w} / \mathrm{v}$ sodium deoxycholate (SDC), $1 \mathrm{mM}$ tris (2-carboxyethyl) phosphine (TCEP), and $2.5 \mathrm{mM}$ 2-chloroacetamide (CAM). For the PBS-high salt fraction, 30ug protein was added to 1 in 5 parts of $20 \%$ w/v Trichloroacetic acid (TCA) and $3 \times$ volume of acetone, and left to incubate on ice for $1 \mathrm{~h}$. Following centrifugation at $14,000 \mathrm{~g}$ for $1.5 \mathrm{~min}$ at room temperature, pelleted samples were washed with $200 \mathrm{ul}$ of acetone and pelleted material brought up in $20 \mathrm{ul} \mathrm{MRAB}$. For the membrane protein pellet fraction, 30 ug protein was added to $20 \%$ of $100 \% \mathrm{w} / \mathrm{v} \mathrm{TCA}$, and left to incubate on ice for $1 \mathrm{~h}$. Following centrifugation at $14,000 \mathrm{~g}$ for $1.5 \mathrm{~min}$ at room temperature, pelleted samples were also washed with $200 \mathrm{ul}$ of acetone and pelleted material resolubilized in
$20 \mathrm{ul}$ of MRAB. Validation of protein separation in all three protein fractions was conducted using sypro-red and Coomassie staining for total protein after polyacrylamide gel electrophoresis. Seven and a half microliters of all three protein fractions underwent trypsin digestion at a 1:100 enzymatic concentration. Firstly re-suspended samples in MRAB were incubated at $37^{\circ} \mathrm{C}$ for $30 \mathrm{~min}$; $7.5 \mathrm{ul}$ of prepared activated trypsin solution (Promega, WI, USA) was added to re-suspended samples, and further incubated overnight at $37{ }^{\circ} \mathrm{C}$ while shaking mildly. Digested samples were stored at $-80{ }^{\circ} \mathrm{C}$ prior to TMT labeling.

\section{Tandem mass tag (TMT) labeling strategy}

We used a multiplexed isobaric labeling approach to allow for simultaneous identification and quantification of proteins from multiple biological samples. A 10-plex TMT labeling kit (ThermoScientific, NJ, USA) was used for analyses of protein samples from controls and AD with different APOE genotypes, with the control E3/E3 used as a reference sample per plex for normalization of data and as a reference point for the different runs. This labeling strategy allowed for all different groups (i.e. disease and genotype) to be randomized and analyzed within the same batch pool. All samples and isobaric label tags were handled blind to the experimenter. Twenty microliter aliquots of each label (dissolved in $20 \mathrm{ul}$ of acetonitrile solution) were dried down in the speed vacuum and re-suspended in $25 \mathrm{mM}$ TEAB made up in acetonitrile solution. Re-suspended labels were subsequently added to $10 \mathrm{ul}$ of dried digested protein samples, and allowed to incubate for $1 \mathrm{~h}$ at room temperature, after which $1 \mathrm{ul}$ of formic acid solution was added to stop the reaction. Labeled samples were pooled together in entire batches and subsequently dried in the speed vacuum. All three subcellular fractions were processed and this consisted of 18 batches of different 10-plex TMT pools.

\section{Sodium deoxycholate (SDC) and tetraethylammonium bromide (TEAB) clean up}

To remove traces of SDC and TEAB, protein samples were re-suspended in $100 \mathrm{ul}$ of $1 \%$ formic acid solution

\footnotetext{
(See figure on next page.)

Fig. 1 Summary of liquid chromatography/mass spectrometry (LC/MS) and proteomic analyses of tissue from the cerebrovasculature isolated from the inferior frontal gyrus in Alzheimer's disease (AD) and healthy control cases from different APOE genotypes. (A) shows brain region of interest used to isolate cerebrovessel fractions. (B) shows identified total number of quantified spectra, peptide spectrum matches and non-redundant master protein groups from all TMT experiments. (C) Data shows level of expression for gene IDs associated with specific cell types identified in our proteomic analyses of the isolated cerebrovasculature (i.e. astrocytes, microglia, pericytes, endothelial cells, and smooth muscle cells). Data represent ratio expressed in arbitrary units. Venn diagram shows unique and overlapping significantly regulated proteins in the comparisons between (D) healthy controls from APOE2/2 vs APOE3/3, APOE3/4 vs APOE3/3, and APOE4/4 vs APOE3/3 genotypes, (E) Healthy matched Alzheimer's disease cases vs controls from APOE2/3, APOE3/3, APOE3/4, and APOE4/4 genotypes. Asterisk in Venn diagram [*] denote unique non-overlapping proteins from each comparisons. F, G and $\mathbf{H}$ shows the violin plot for Amyloid plaque score, Tangle score and CAA score, respectively
} 


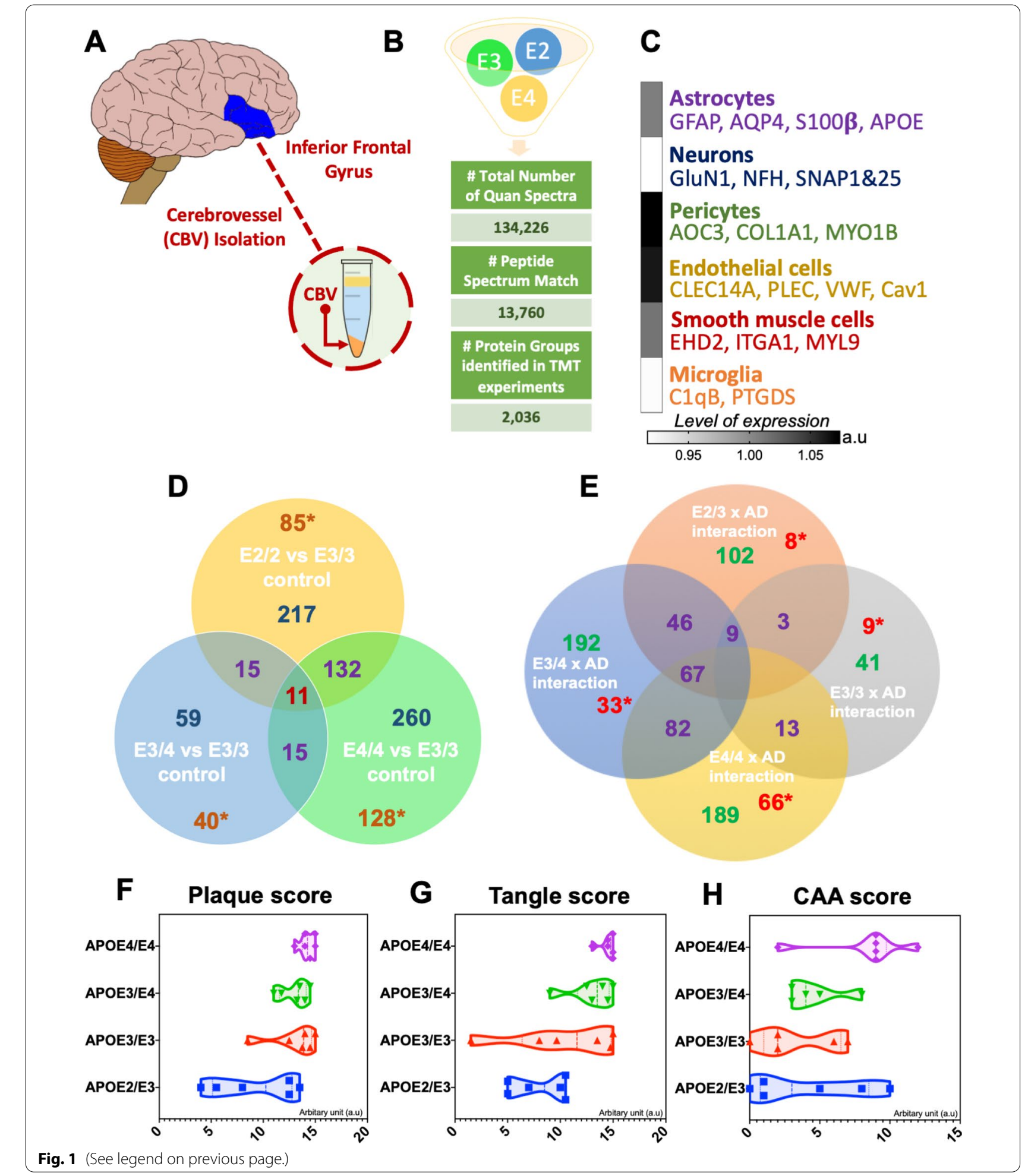

C

and centrifuged at 15,000 rpm for 1 min to allow separation into different phases. Supernatants were collected in new Eppendorf tubes, and 200ul of ethyl acetate was added, and centrifuged at 15,000 rpm with the upper organic layer discarded. This process was repeated three separate times, with the final lower phase taken to dryness in the speed vacuum. The resultant dried samples were re-solubilized in $100 \mathrm{ul}$ of $0.1 \%$ formic acid. 


\section{Purification and concentration of peptides}

Prior to ultra-high pressure liquid chromatography (UHPLC), single step desalting, concentration and purification of peptides were conducted using $0.6 \mathrm{ul} \mathrm{C18}$ resin Ziptips (ThermoScientific, NJ, USA). Briefly, ziptips pipette tips were used to remove contaminants by aspirating and dispensing in a solution of $0.1 \%$ formic acid made up in 50\% acetonitrile (i.e. wetting buffer), and afterwards in a solution containing $0.1 \%$ formic acid (i.e. binding buffer). Ziptips were used for sample binding, by aspirating and dispensing through the samples multiple times. The resultant concentrated and purified labeled samples were aspirated in a solution of $5 \%$ methanol and $0.1 \%$ formic acid (i.e. washing buffer), followed by elution in a solvent containing $10 \mathrm{ul}$ of $0.1 \%$ formic acid made up in 50\% acetonitrile (wetting buffer). After desalting and concentrating peptides, final samples were dried and re-suspended in $20 \mathrm{ul}$ of $0.1 \%$ formic acid and subsequently transferred into an auto-sampler vial, and analyzed by nano-Ultra-Performance Liquid Chromatography (UPLC) MS on a Q-Exactive Orbitrap instrument (ThermoScientific, NJ, USA).

\section{Chromatography and mass spectrometry (LC-MS/MS) methods}

Pooled TMT-labeled peptides were analyzed using LCMS/MS (Q-Exactive). A Thermo Easy UPLC was operated in a vented trap/elute configuration to separate TMT-labeled peptides. $5 \mu$ l of each re-constituted sample was loaded onto a $0.075 \times 20 \mathrm{~mm}$ Pepmap C18 trapping column at $98 \%$ mobile phase A (MPA, $0.1 \%$ formic acid, water), and diverted to a $0.075 \times 500 \mathrm{~mm}$ C18 Pepmap reversed phase column ( $2.0 \mu \mathrm{m}$ particle) following an isocratic loading and washing step. Peptides were separated over a $4.5 \mathrm{~h}$ linear gradient of increasing mobile phase B (MPB, $0.1 \%$ formic acid, 99.9\% acetonitrile) from 2 to 30 percent at $250 \mathrm{nl} / \mathrm{min}$ and $50{ }^{\circ} \mathrm{C}$. Xcalibur (Thermo) software was used to control the instrument in data dependent acquisition (DDA) mode. DDA settings for these MS experiments followed our previous work [57, 61], and were as follows: full-scan MS resolution $=140$ 000 full width at half maximum at $200 \mathrm{~m} / \mathrm{z}$, full-scan range $=380-1250 \mathrm{~m} / \mathrm{z}$, isolation width $=1.2 \mathrm{~m} / \mathrm{z}$, higher energy C-trap dissociation relative collision energy $=29$, a minimum $\mathrm{m} / \mathrm{z}$ setting of $100 \mathrm{~m} / \mathrm{z}$ was used for all $\mathrm{MS}^{2}$ spectra, $\mathrm{MS}^{2}$ resolution $=35,000$, dynamic exclusion $=180 \mathrm{~s}$, and a Top 15 high/low duty cycle was used for precursor ion selection. Sample limitations did not allow for extensive two-dimensional (2D) fractionation of the labeled peptides, therefore we the use of a narrow isolation window and an ultra-long shotgun gradient was used to minimize the deleterious effects on quantitative accuracy that typically result from co-isolation of isobaric precursors.

\section{Data processing and statistical analysis of proteomics data} We surveyed our amalgamated data-files, and added other modifications to our search criteria if deemed necessary, using the PMi preview software. Preview results were used to choose the precursor and fragment ion mass tolerances (4-ppm, 0.02-Da, respectively) and dynamic modifications. We used the following settings to search the data using SEQUEST and BYONIC as the search algorithms, and Uniprot human database (FEB/2018). Dynamic modifications-Oxidation/+15.995 Da (M), Methyl/+14.016 Da (E), Deamidated/+0.984 Da (N, Q), static modifications of TMT 10-plex/+229.163 Da (N-Terminus, K), Carbamidomethyl+57.021 (C). A coisolation filter within PD 2.1 was set to $50 \%$ to exclude those PSMs which arose from MS/MS spectra that arose from co-isolation of precursor ions as a means to minimize the effects of signal distortion in the reporter ion region inherent to chimeric MS/MS. Only unique peptides were considered for our final quantification. We used the Percolator feature of Proteome Discoverer for SEQUEST, and used the target-decoy feature, to set a false discovery rate (FDR) of 0.01 for Byonic. The peptides passing this stringent cutoff FDR rate were subsequently exported for data cleaning and statistical analysis in JMP (SAS) software (version 15). Master proteins only underwent quantitative analysis if they were identified in at least $50 \%$ of the total number of plexes. JMP software employs a Shapiro-Wilk test for normality which was assessed prior to statistical analyses. Raw ion counts were $\ln$ transformed and analyzed by either two-way analyses of variance (ANOVA) (for AD and APOE interactions) or t-test (Control E2/E2 vs E3/E3, E3/E4 vs E3/ E3, E4/E4 vs E3/E3 comparisons) to interrogate significantly regulated proteins between groups. Abundance ratio was generated by dividing each samples with the respective control E3/E3 sample used as a reference in the same plex for normalization of data (see Table 1). Log2 fold change and negative log 10 of the $p$ value were subsequently uploaded into ingenuity pathway analyses (IPA) where molecules and pathways, diseases and biofunctions, associated networks and upstream regulators unique to each group comparison(s) were identified. Only master proteins with a significance level of 1.3 (i.e. negative $\log 10$ of $\mathrm{p}$ value) were uploaded into IPA. We have deposited the mass spectrometry proteomic data into the ProteomeXchange Consortium via the PRIDE partner repository [62]. Our datasets can be located with the unique identifier-PXD023340. 


\section{Ingenuity pathway analysis}

All datasets of significantly modulated proteins from our group comparisons were uploaded into the Ingenuity Pathway Analysis software (IPA, Ingenuity ${ }^{\circledR}$ Systems [63]) to map them onto known networks of protein interactions in the knowledgebase. We further used the IPA knowledgebase to further determine the significantly regulated Canonical pathways/disease and biofunctions and biological significance of APOE and AD-dependent changes in the cerebrovasculature. Our core analysis settings involved the following-Ingenuity Knowledge base as reference set, maximum number of 35 molecules per network, and a maximum number of 25 networks for analysis. Only experimentally observed knowledge was considered in our analyses. We controlled for data sources, species, and tissue type/cell lines at the time of analysis in IPA. Core analysis identified canonical pathways shown to be significantly altered in response to APOE genotype and AD pathogenesis as a result of significantly regulated proteins represented in those pathways/ biofunctions. Statistical significance of the relationship between uploaded dataset and the identified pathways/ biofunctions was measured using two methods: (1) Ratio of the number of molecules from the data set that map to a pathway/biofunction divided by the total number of molecules in that pathway/ biofunction knowledgebase in IPA. (2) Fisher's exact test, to calculate a p-value determining the probability that the association between the proteins in the dataset and the pathway/biofunctions are explained by chance alone. $\mathrm{P}$ values were considered to be significant in these studies when $P<0.01$. Upstream regulator analysis was used to predict the upstream transcriptional master regulators in our proteomic dataset, and this was generated using the Ingenuity ${ }^{\circledR}$ Knowledge Base. An overlap P value was generated based on analyses of the significant overlap between proteins/genes in our dataset and known targets modulated by the transcriptional regulator or Upstream master regulator. The activation z-score algorithm was used to make predictions.

\section{Results}

\section{Demographics and clinical background of patient population}

In this study, we used 54 total brain cerebrovascular specimens from the inferior frontal gyrus of healthy controls (29) and Alzheimer's disease (AD) patients (26 cases)(see Table 1). Both control and AD cases consisted of five different APOE genotypes, APOE2/2, APOE2/3, APOE3/3, APOE3/4, and APOE4/4. APOE2/2 cases were low in sample size for the AD group, and as such were removed from our analyses. On average control patients $(72.9 \pm 3.49)$ were younger than AD cases $(85.24 \pm 2.10)$. This was driven by the younger age of APOE4/4 controls
$(45.83 \pm 4.39)$. Most of the other cases were comprised of septuagenarians and octogenarians. Each group consisted of mixed genders, and on average, there were more females in both controls (62.1\%) and AD (61.5\%) cohorts.

Alzheimer's disease cases were classified based on the Braak staging, amyloid plaque score, and Tangle score. The majority of AD cases consisted of Braak stage IVVI, Tangle score ranging from 8 to 15 , and plaque score between 7 and 14 (Table 2). E4/E4 cases had the worst Braak staging, and plaque score (Table 2; see also Fig. 1F and $1 G)$. E4/E4 also had the highest mean CAA score (8), while E3/E3 had the lowest score (Table 2; see also Fig. 1H). E3/E3 and E4/E4 AD groups had the worst mean final MMSE scores (6 and 11 respectively), and the E2/E3 and E3/E4 (both approx.18) had the highest mean final MMSE scores (Table 2). E4/E4 AD patients also had the smallest brain weight $(959 \pm 18.1)$ of all cases (Table 2$)$.

\section{Proteomic profiles, cell type changes and altered canonical pathways in enriched cerebrovascular tissue from the inferior frontal gyrus of control patients from different APOE genotypes}

A 10-plex TMT isobaric tag approach was used to study the proteomic profiles of brain cerebrovascular tissue from the inferior frontal gyrus of $\mathrm{AD}$ cases and controls. We identified a total of 13,760 total peptide spectrum matches and 2036 non-redundant master protein groups from all TMT experiments (Fig. 1B). To determine the cell type constituents of our enriched cerebrovascular tissue we identified cell specific protein markers in our proteomic dataset using the single cell sequencing resource from the PanglaoDB omic database. We measured the relative protein expression levels of these specific markers associated with these different cell types in control samples, and observed that there was a relatively high expression and abundance levels of markers associated with pericytes (AOC3, COL1A1, MYO1B) and endothelial cells (CLEC14A, VWF, Cav1, PLEC) in our enriched cerebrovascular fractions (Fig. 1C). This was followed by smooth muscle cells (EHD2, ITGA1, MYL9) and astrocytes (GFAP, AQP4, S100 $\beta$, APOE). Neurons and microglia related proteins were least expressed in our cerebrovascular fractions.

Prior to comparing the disease and APOE genotype interaction effects in our proteomic analyses, we first interrogated any underlying APOE genotype effects in the healthy controls that may drive disease outcome in $\mathrm{AD}$ patients. We focused this analyses on healthy control cohorts from E2/E2, E3/E3, E3/E4 and E4/E4 groups. A T-test approach was used to analyze the master proteins to identify significant changes in unique and common proteins altered between APOE $4 / 4$ vs APOE3/3, APOE3/E4 vs APOE3/E3, and APOE2/2 vs 


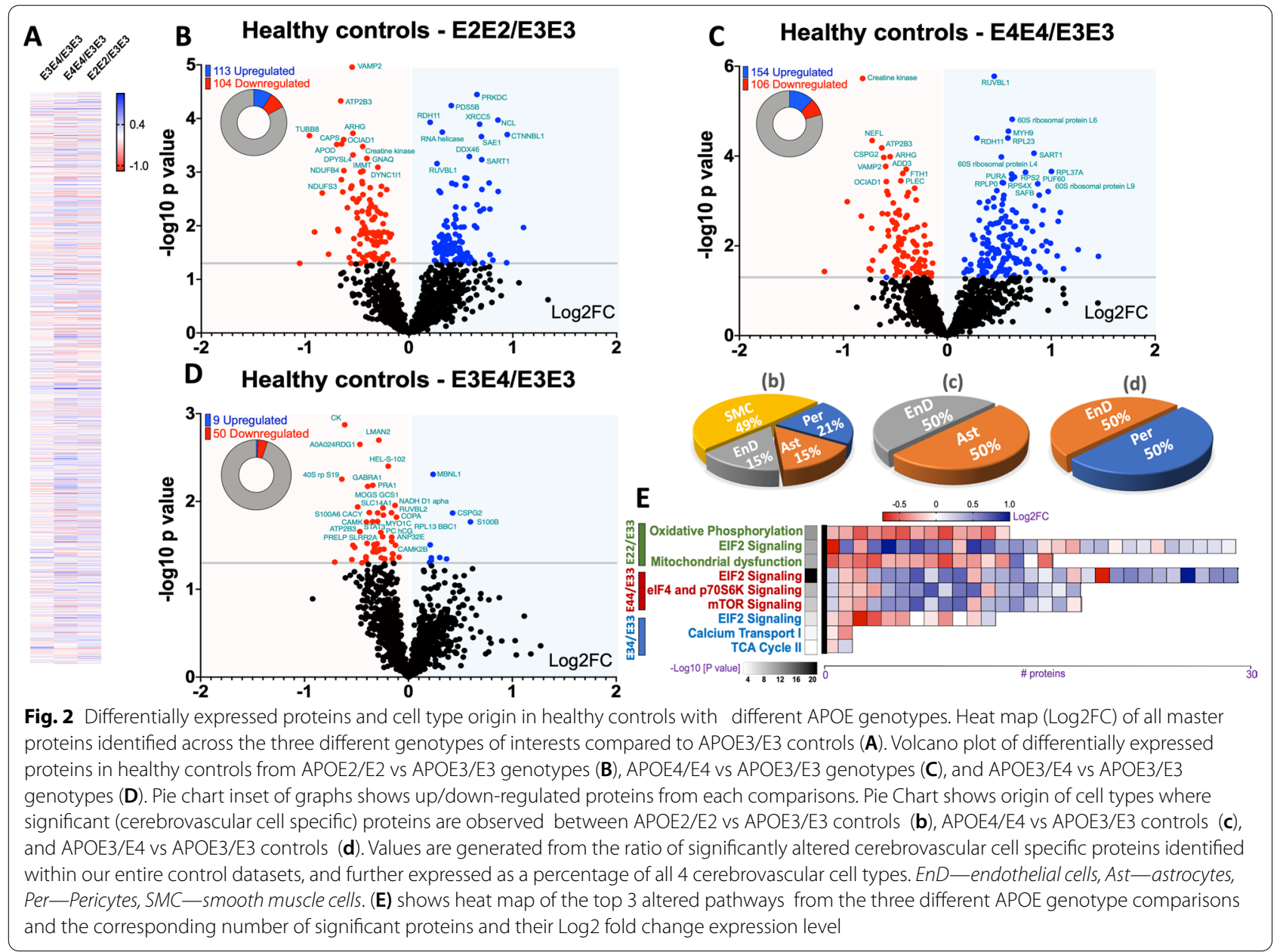

APOE3/3 genotypes. Statistical analyses identified a total of 217 master proteins significantly changing in APOE2/2 vs APOE3/3, 260 in APOE4/4 vs APOE3/3, and 59 in APOE3/4 vs APOE3/3 (Fig. 1D-for a full list of significantly regulated proteins, see Additional files 1, 2, 3: Tables S1-S3). Heat map of all proteins identified, and the distribution of $\log 2$ fold change in correlation with negative $\log 10 \mathrm{p}$ value can be found in Fig. 2A-D.

We interrogated the origin of cell types showing the most significant changes in cerebrovascular cell specific markers within our control datasets, and expressed this as a percentage of all 4 cerebrovascular cell types. We observed that most of the significant changes in our proteomic datasets between APOE2/2 vs APOE3/3 controls were associated with endothelial cell $(15 \%)$, pericyte $(21 \%)$, astrocyte $(15 \%)$, smooth muscle cell specific proteins (49\%) (Fig. 2b). We also revealed that most of the significant changes in our proteomic datasets between APOE4/4 vs APOE3/3 controls were associated with endothelial cell (50\%) and astrocyte specific proteins (50\%) (Fig. 2c). While significant changes in cell specific markers between APOE $3 / 4$ vs APOE $3 / 3$ controls were mainly associated with endothelial cell $(50 \%)$ and pericyte specific proteins (50\%) (Fig. 2d).

We categorized the significant proteins identified above into their subcellular origin, and functional sub-groups (i.e. enzymes, receptors, transporters etc.). We noted a fairly similar change across the three different genotype group comparisons (see Fig. 3). Significantly regulated proteins were typically of cytoplasmic and nuclear origins (Fig. 3A), and were primarily enzymes, transcription regulators and transport/carrier proteins (Fig. 3B).

Ingenuity pathway analyses revealed numerous pathways significantly impacted within control groups from different APOE genotype (Fig. 4). The top 3 pathways altered in APOE4/4 vs APOE3/3 controls include downregulation of EIF2 signaling, regulation of EIF4 and p70S6k signaling, and mTOR signaling. Top 3 altered pathways in APOE2/2 vs APOE3/3 controls were oxidative phosphorylation, EIF2 signaling 

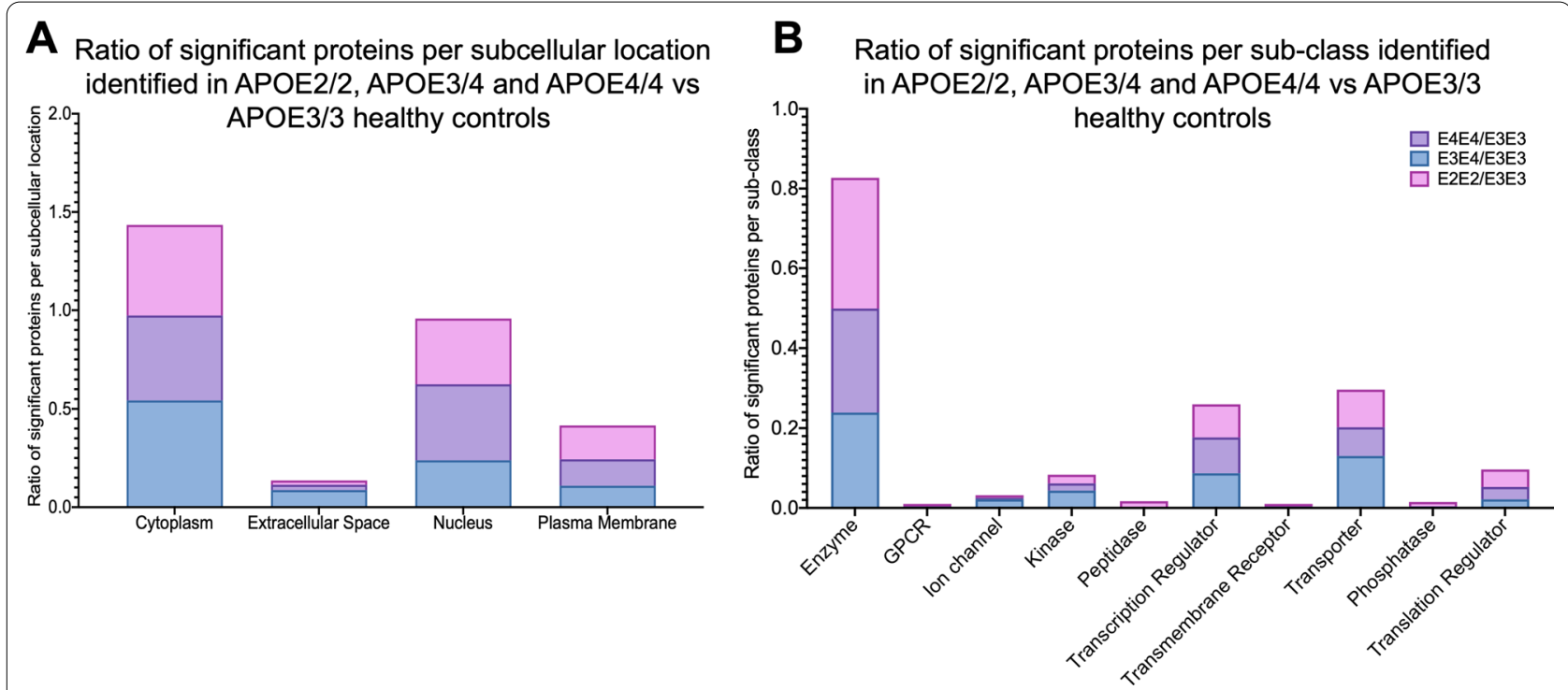

Fig. 3 Subcellular localization and sub-class of proteins identified in the cerebrovessels of the inferior frontal gyrus in APOE2/2, APOE3/4 and APOE4/4 vs APOE3/E3 healthy controls. A shows ratio of significant proteins localized to the cytoplasm, extracellular space, nucleus and plasma membranes. B shows ratio of significant proteins and their corresponding sub-classification

and mitochondrial dysfunction. While in APOE3/4 vs APOE3/3 control, downregulation of EIF2 signaling, altered Calcium transport and TCA cycle were the top 3 pathways signficantly altered. Heat map in Fig. 2E, shows the number of proteins associated with the top 3 pathways, and their direction of change.

Of the significantly regulated proteins identified from our APOE genotype comparisons, we identified 85 out of 217 to be unique to the APOE2/2 vs APOE3/3 group alone, 128 out of 260 to the APOE4/4 vs APOE3/3 group, and 40 out of 59 proteins to the APOE3/4 vs APOE3/3 group (see Venn diagram-Fig. 1D). We used the ingenuity pathway analyses to identify the pathways unique to only APOE2/2 vs APOE3/3, these included mitochondrial dysfunction, chemokine signaling, fMLP signaling in immune cells, Phospholipase C, GNRH and CCR3 signaling (Fig. 4). We identified TCA cycle, Calcium transport I, HIF $\alpha$ signaling and oxidized GTP and dGTP detoxification as pathways unique to only the APOE3/4 vs APOE3/3 genotype comparison (Fig. 4). While semaphorin A signaling and granzyme A signaling were the only pathways unique to the APOE4/4 vs APOE3/3 comparison (Fig. 4).
Our interrogation of APOE levels in the cerebrovascular fractions revealed no significant changes across the genotypes in healthy non-demented cases (Fig. 5).

\section{Proteomic profiles, cell type changes and canonical pathways altered in cerebrovascular tissue from the inferior frontal gyrus of control vs $A D$ cases from different APOE genotypes}

A two way ANOVA approach was used to identify significant changes in master proteins showing a genotype and disease interaction. Statistical analyses identified a total of 102 master proteins in the E2/E3 AD cases vs controls, 41 proteins in the E3/E3 AD cases vs controls, 192 proteins in the E3/E4 cases vs controls, and 189 proteins in the E4/E4 cases vs controls (Fig. 1E-for a full list of significantly regulated proteins see Additional files 4, 5, 6, 7: Tables S4-S7). A list of the Top 25 proteins signficantly altered in E3/E4 and E4/E4 AD cases vs controls are provided (see Tables 4 and 5). Heat map of all proteins identified, and the related volcano plots can be found in Fig. 6A-D.

We interrogated the origin of cell types showing the most significant changes in cerebrovascular cell specific markers within our $\mathrm{AD}$ vs control datasets to

(See figure on next page.)

Fig. 4 Canonical pathways modulated in the cerebrovasculature of the inferior frontal gyrus in healthy controls with different APOE genotypes cases. Identified canonical pathways were generated from the list of significantly modulated proteins between healthy controls from APOE2/E2 vs APOE3/E3, APOE3/E4 vs APOE3/E3, and APOE4/E4 vs APOE3/E3 genotypes using Ingenuity pathway analyses. Values represent negative log 10 of FDR adjusted $P$ value after Fischer's test and Benjamin Hochberg correction. Significant cut-off is set at $\geq 2$. APOE2/E2 vs APOE3/E3 (45 pathways identified), APOE4/E4 vs APOE3/E3 (25 pathways identified), and APOE3/E4 vs APOE3/E3 (7 pathways identified) 


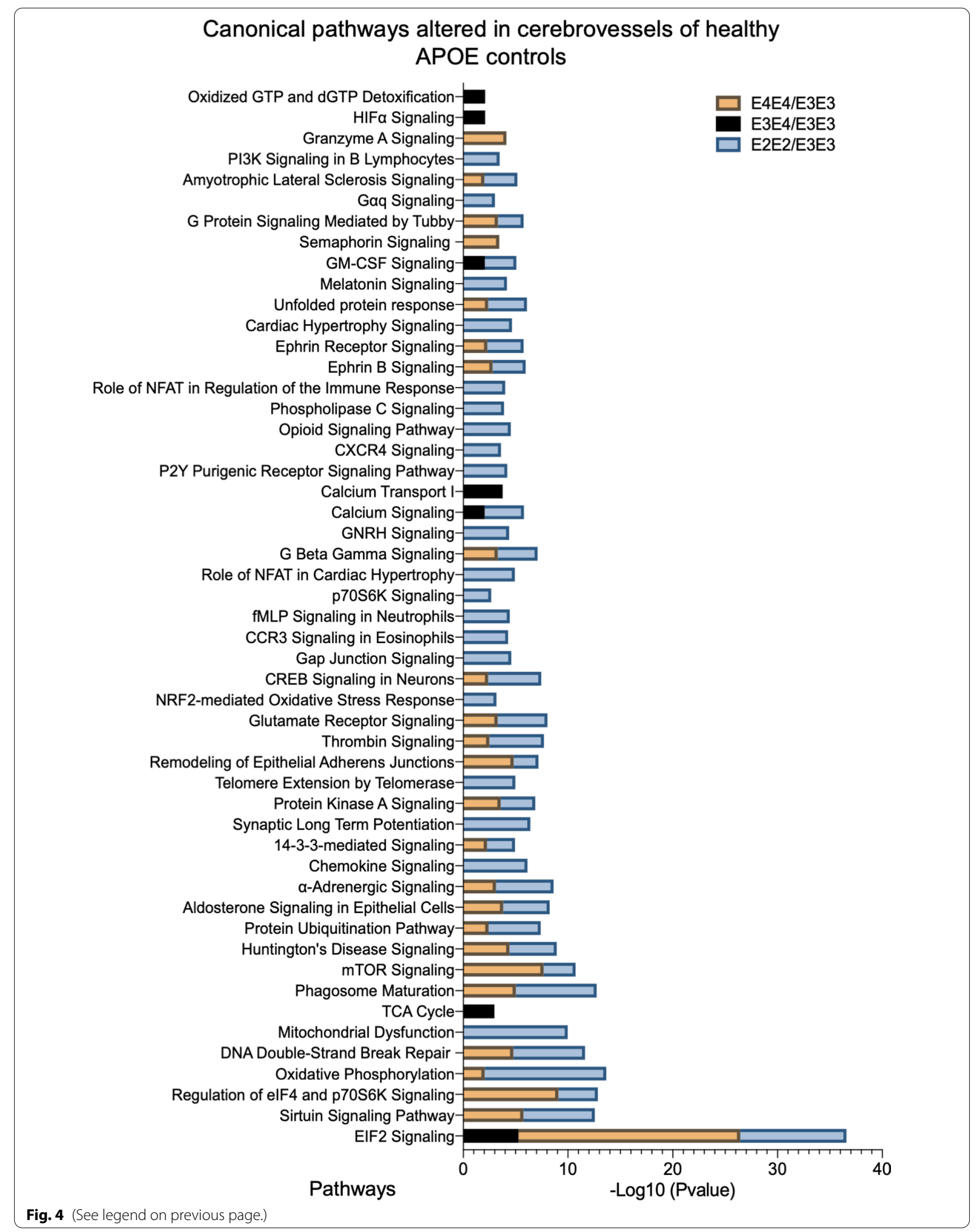




\section{Cerebrovascular APOE Levels}

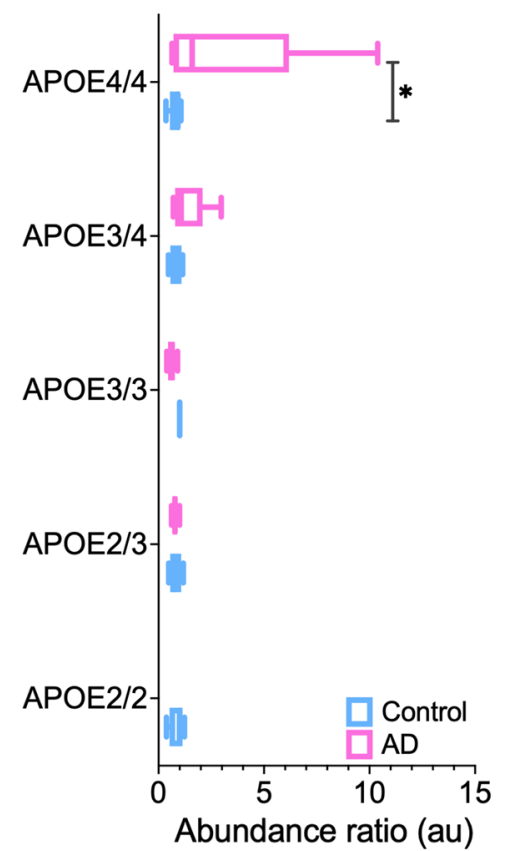

Fig. 5 APOE levels in the cerebrovasculature of the inferior frontal gyrus in AD cases vs healthy controls from different APOE genotypes. Asterisk denotes ${ }^{*} \mathrm{P}<0.05$

demonstrate the impact of our proteomic changes at the single cell level (Fig. 6b-e). We observed that the origin of cell specific protein markers demonstrating significant changes in $\mathrm{E} 2 / \mathrm{E} 3 \mathrm{AD}$ cases vs controls were derived primarily from astrocytes (100\%) (Fig. 6b). We observed a similar outcome in E3/E3 cases vs controls (Fig. 6c). In the E3/E4 cases, we observed changes in-smooth muscle cell derived proteins (44\%), astrocytes and endothelial cell specific proteins (each 28\%) (Fig. 6d). While in the E4/E4 cases, we observed changes implicating 2 cell types, with $50 \%$ attributed to astrocytes and $50 \%$ to endothelial cells (Fig. 6e).

We stratified the significant proteins identified from the disease $x$ genotype interactions into their subcellular origins and functional sub-groups. Significantly regulated proteins identified between AD vs controls from E2/3 and E4/4 genotypes were primarily localized to the nucleus, while E3/3 cases were localized to the cytoplasm (Fig. 7A). With respect to the functional sub-groups, significantly regulated proteins identified between $A D$ vs control cases from E2/3, E3/4 and E4/4 genotypes were primarily enzyme-related proteins and transcriptional regulator proteins, while E3/3 cases consisted of mainly enzyme-related and transport/carrier proteins (Fig. 7B).
Ingenuity pathway analyses of significantly regulated proteins revealed numerous pathways impacted by disease and APOE genotype interactions (Table 3). The Top 3 pathways identified between $\mathrm{AD}$ vs controls from E3/ $\mathrm{E} 4$ and E4/E4 genotypes include EIF2 signaling, regulation of EIF4 and p70S6k signaling and mTOR signaling. In the E3/E3 AD vs control groups, we identified EIF2 signaling, mTOR signaling and Glutamine Biosynthesis I as the top 3 pathways. While from the E2/E2 AD vs control groups the Top 3 pathways identified were spliceosomal cycle, DNA methylation and transcriptional repression signaling and Base excision repair (BER) pathways involved in the repair of damaged DNA during the cell cycle. Heat map in Fig. 6F, shows the number of proteins associated with the top 3 significantly modulated pathways from the four different disease $\times$ APOE genotype interactions described above, and their direction of change.

Of the significantly regulated proteins showing a disease and APOE genotype interaction, we identified only 8 out of 102 to be unique to the APOE2/3 group alone, 9 out of 41 to the APOE3/3 group, 33 out of 192 to the APOE3/4 group, and 66 out of 189 proteins to the APOE4/4 group (see Venn diagram-Fig. 1E). Ingenuity pathway analyses generated pathways unique to disease and (E4/E4) genotype interaction alone, some of which included Tight junction signaling, neucleobases (pyramidine) synthesis, actin cytoskeleton signaling and Integrin linked kinase signaling, and from the E3/E4 group the unique pathways identified were sirtuin signaling pathway, remodeling of adheren junctions, Telemore extension, 14-3-3 mediated signaling, Caveolae mediated endocytosis signaling and CCR3 signaling (Table 3). CXCR4 signaling, NRF-2 mediated oxidative stress response, Thrombin, and aldosterone signaling were some of the top pathways unique to the E3/E3 group (Table 3). Only DNA methylation and transcriptional repression signaling pathway were unique to the disease and (E2/E3) genotype interaction (Table 3).

Our assessment of APOE levels in the cerebrovascular tissue showed a significant increase in AD vs controls from the APOE4/4 genotype, and a trend towards increase in APOE3/4 cases (Fig. 5).

We interrogated the Top 5 Upstream Regulators mediating the changes observed between disease $\mathrm{x}$ APOE genotype interactions (Fig. 8). From the E2/E3 group, IPA identified a significant overlap in our dataset and known targets regulated by these five Upstream regulators, MMP3 (matrix metalloproteinase protein 3), CST5 (cystatin-D), 5-Fluorouracil (pyrimidine analogue blocking DNA synthesis), MYC (Proto-Oncogene, BHLH Transcription Factor), tanespimycin (HSP90 inhibitor) (Fig. 8A). From the E3/E3 group we revealed MAPT 


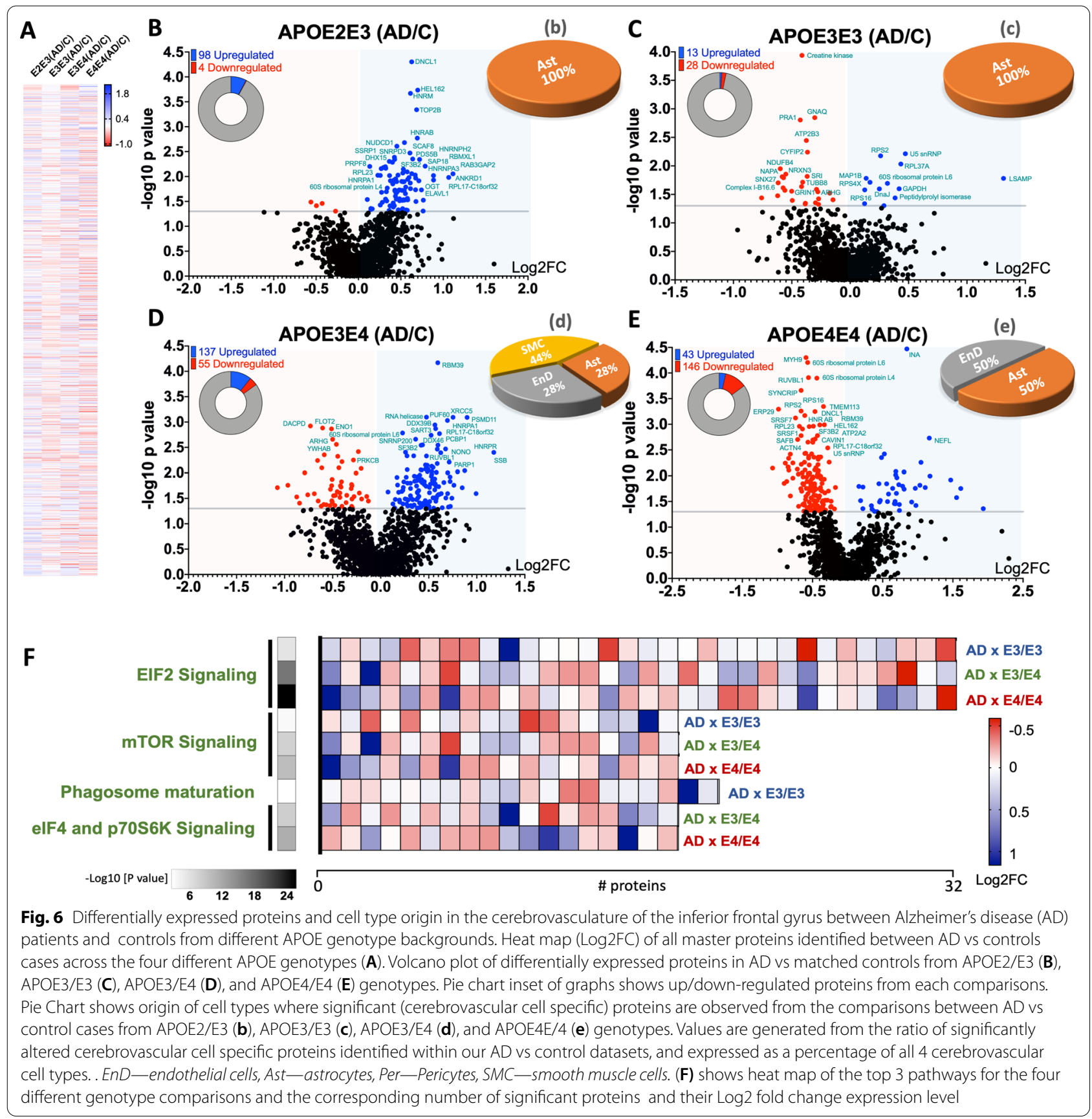

(microtubule associated protein tau), MYCN (N-myc Proto-Oncogene, BHLH Transcription Factor), DPP23 (apoptosis activator IX), BDNF (brain derived neurotrophic factor), and HTT (huntingtin) as the top 5 upstream regulators (Fig. 8B), while MYC, RICTOR (rapamycin-insensitive companion of mammalian target of rapamycin), MAPT, 5-fluorouracil, and MMP3 were identified in the E3/E4 group (Fig. 8C). MYCN, rapamycin (mTOR inhibitor), 5-fluorouracil, MYC, and MMP3 were also identified as top upstream regulators in the E4/ E4 groups (Fig. 8D) (Tables 4, 5).

\section{Discussion}

Very few studies have explored the influence of APOE genotype on the pathobiological changes to the cerebrovasculature in the pathogenesis of $\mathrm{AD}$. We hypothesized that APOE genotype specific molecular aberrations (at the protein level) in the cerebrovasculature, may 


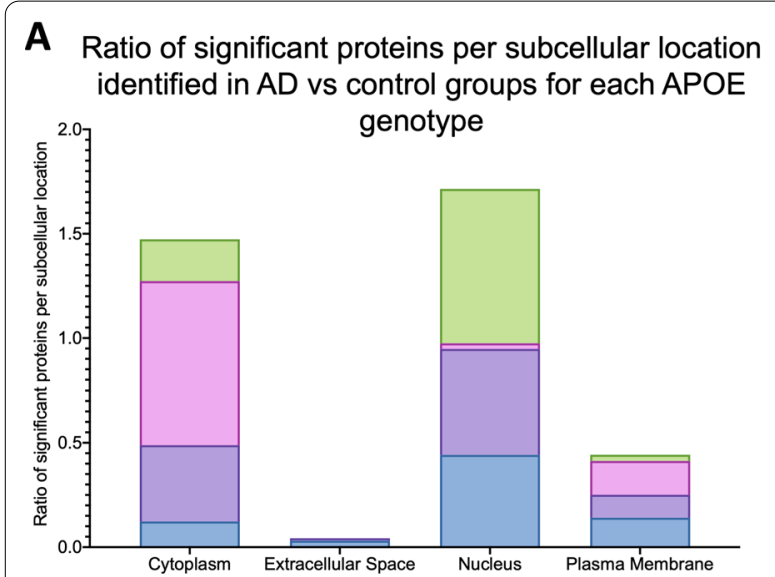

\section{B Ratio of significant proteins per sub-class identified in $A D$ vs control groups for each APOE genotype}

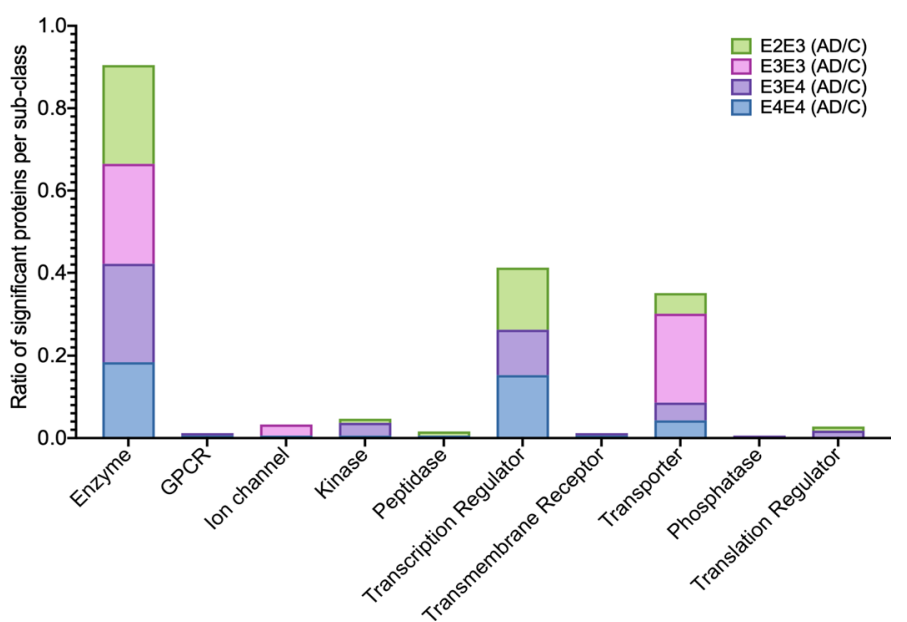

Fig. 7 Subcellular localization and sub-class of proteins identified in the cerebrovessels of the inferior frontal gyrus in AD cases vs controls from APOE2/E3, APOE3/E3, APOE3/E4 and APOE4/E4 genotypes. (A) shows ratio of significant proteins localized to the cytoplasm, extracellular space, nucleus and plasma membranes. (B) shows ratio of significant proteins and their corresponding sub-classification

compromise their physiological properties and essential functions, and thus explain the influence of APOE genotype on cerebrovascular dysfunction in AD pathogenesis.

To address this, we employed our state-of-the-art proteomic platform to conduct a detailed unbiased characterization of changes in protein expression levels, and molecular pathways significantly altered in cerebrovessels isolated from the inferior frontal gyrus of control and $A D$ brains from five different APOE genotype backgrounds. The cerebrovessel fraction expressed very high levels of endothelia and pericyte cell specific markers, and a moderate expression level of astrocyte and smooth muscle cell specific markers. From our proteomic analyses we revealed unique changes in proteins and molecular pathways driven by APOE genotype that could explain the vulnerability of the cerebrovasculature in the pathological sequelae of AD.

\section{APOE specific effects in non-demented cases}

The insidious cascades of events triggered in the brains of Alzheimer's disease patients is thought to have begun many years before the onset of symptoms. Therefore, we first set out to investigate the underlying changes in healthy control APOE genotypes (i.e. E2/ $\mathrm{E} 2, \mathrm{E} 3 / \mathrm{E} 3$, and E4/E4) to determine whether underlying isoform specific differences exist in non-demented patients that could be prodromal to and contribute to $\mathrm{AD}$ pathogenesis. As E3/E3 is the most common genotype, we used the control E3/E3 cases as the normal (reference) phenotype in our comparisons. Moreover the use of homozygote genotypes ensured a cleaner analyses of the three different alleles/isoforms in isolation without the confounding influence in heterozygote genotypes. It is worthy of note that the mean age of E2/E2 patients we analyzed was $71 \mathrm{yrs}$, while E3/ E3 were slightly older, approximately 83yrs. Due to the scarcity of aged healthy control non-demented E4/ E4 patients, we were only able to obtain samples from younger cohorts with a mean age of 45 yrs, thus this age bias has to be factored into the discussion of our results as a limitation of this study. The clinical background of the E2/E2 and E3/E3 controls demonstrated similar MMSE scores (28-29), low Braak staging (II) and plaque score (1-3). Due to the relatively young mean age of E4/E4 controls (most died as a result of natural causes unrelating to brain disorders) there was no detailed neuropathological examination conducted, thus we do not have information on their cognitive status or tau/amyloid pathology scores.

We observed 217 significantly regulated proteins in E2/ E2 vs E3/E3 controls and Ingenuity pathway analyses identified over 40 pathways. Notable changes included, EIF2 signaling, oxidative phosphorylation, mitochondrial dysfunction, phagosome maturation, chemokine and sirtuin signaling and DNA repair mechanisms. Of the 40 pathways significantly altered, 15 were unique to the E2/E2 vs E3/E3 comparison alone, with the most significant of these being mitochondrial dysfunction and Chemokine signaling. Thus indicating that the E2 allele/ isoform may have specific roles in contributing towards 
Table 3 Canonical pathways modulated in the cerebrovasculature of the inferior frontal gyrus between Alzheimer's disease (AD) patients and controls from different APOE genotype backgrounds

\begin{tabular}{|c|c|c|c|c|}
\hline \multirow[b]{2}{*}{ CANONICAL PATHWAYS } & \multicolumn{4}{|c|}{ AD vs Control } \\
\hline & E2/E3 & E3/E3 & E3/E4 & E4/E4 \\
\hline EIF2 Signaling & 2.48 & 5.55 & 16.75 & 25.73 \\
\hline Sirtuin Signaling Pathway & & & 5.13 & \\
\hline Regulation of elF4 and p70S6K Signaling & & 2.53 & 8.02 & 11.39 \\
\hline mTOR Signaling & & 3.32 & 7.77 & 9.86 \\
\hline Remodeling of Epithelial Adherens Junctions & & & 6.93 & \\
\hline Phagosome Maturation & & 2.61 & 4.42 & \\
\hline DNA Double-Strand Break Repair & 2.75 & & 5.34 & 2.23 \\
\hline Telomere Extension by Telomerase & & & 5.21 & \\
\hline Epithelial Adherens Junction Signaling & & & 3.61 & 2.86 \\
\hline Glutamate Receptor Signaling & & 2.31 & & \\
\hline Gap Junction Signaling & & & 3.59 & \\
\hline RhoGDI Signaling & & 2.39 & & \\
\hline Tight Junction Signaling & & & & 3.30 \\
\hline Spliceosomal Cycle & 4.70 & & & 4.16 \\
\hline a-Adrenergic Signaling & & & 2.20 & \\
\hline Thrombin Signaling & & 2.22 & & \\
\hline Calcium Signaling & & 2.20 & & \\
\hline CXCR4 Signaling & & 2.47 & & \\
\hline 14-3-3-mediated Signaling & & & 3.90 & \\
\hline Signaling by Rho Family GTPases & & & & 2.29 \\
\hline Tec Kinase Signaling & & & 2.49 & \\
\hline Pyrimidine Ribonucleotides Interconversion & & & & 3.28 \\
\hline Pyrimidine Ribonucleotides De Novo Biosynthesis & & & & 3.21 \\
\hline Androgen Signaling & & & 2.96 & \\
\hline DNA Base Excition Repair pathway & 2.89 & & & 2.37 \\
\hline Phospholipase C Signaling & & 2.01 & & \\
\hline Actin Cytoskeleton Signaling & & & & 2.53 \\
\hline Integrin Linked Kinase (ILK) Signaling & & & & 2.88 \\
\hline Glycolysis I & & & 2.88 & \\
\hline Cardiac Hypertrophy Signaling & & 2.05 & & \\
\hline Aldosterone Signaling in Epithelial Cells & & 2.45 & & \\
\hline \begin{tabular}{|l|l|} 
DNA Methylation and Transcriptional Repression Signaling \\
\end{tabular} & 3.32 & & & \\
\hline Caveolar-mediated Endocytosis Signaling & & & 2.51 & \\
\hline CCR3 Signaling in Eosinophils & & & 2.31 & \\
\hline NRF2-mediated Oxidative Stress Response & & 2.28 & & \\
\hline ATM Signaling & & & 2.02 & \\
\hline Gluconeogenesis I & & & 2.88 & \\
\hline Glutamine Biosynthesis I & & 2.74 & & 2.08 \\
\hline CCR5 Signaling in Macrophages & & & 2.07 & \\
\hline Sumoylation Pathway & & & 2.05 & \\
\hline Death Receptor Signaling & & & 2.10 & \\
\hline Virus Entry via Endocytic Pathways & & & 2.62 & \\
\hline NADH Repair & & 2.26 & & \\
\hline Granzyme B Signaling & & & 2.11 & \\
\hline Creatine-phosphate Biosynthesis & & 2.04 & & \\
\hline
\end{tabular}


Table 3 (continued)

Identified canonical pathways were generated from the list of significantly modulated proteins between AD vs controls from APOE2/E3, APOE3/E3, APOE3/E4 and APOE4/E4 genotypes using Ingenuity pathway analyses. Values represent negative log 10 of FDR adjusted P value after Fischer's test and Benjamin Hochberg correction. Significant cut-off is set at $\geq 2$. AD vs control APOE2/E3 genotype (5 pathways identified), AD vs control APOE3/E3 genotype (16 pathways identified), AD vs control APOE3/E4 genotype (24 pathways identified), and AD vs control APOE4/E4 genotype (14 pathways identified). Orange highlights-unique to E2/E3 only, Green highlights-unique to E3/E3 only, Blue highlights—unique to E3/E4 only, Red highlights—unique to E4/E4 only

\section{Top upstream regulator}

A

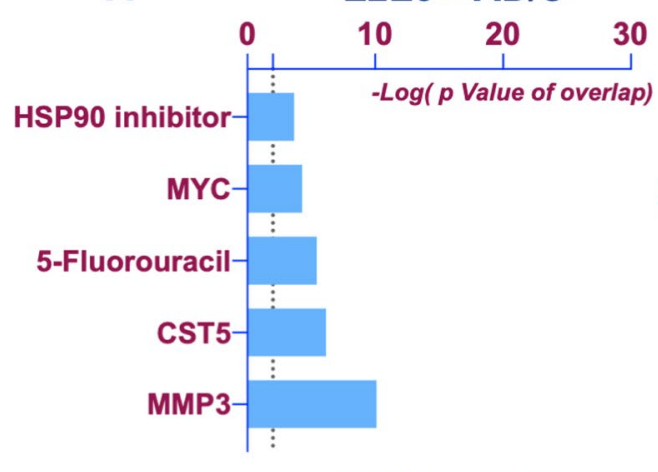

B

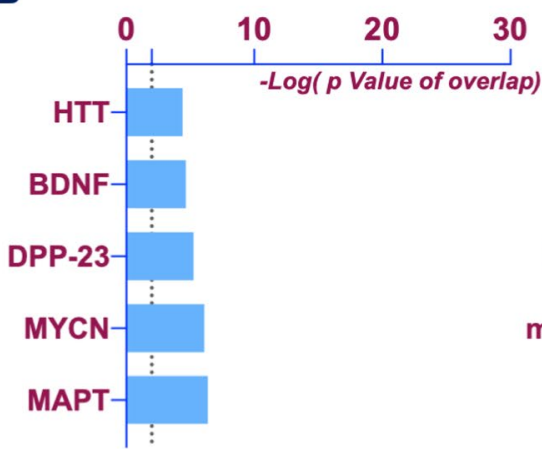

C

E3E4 - AD/C (

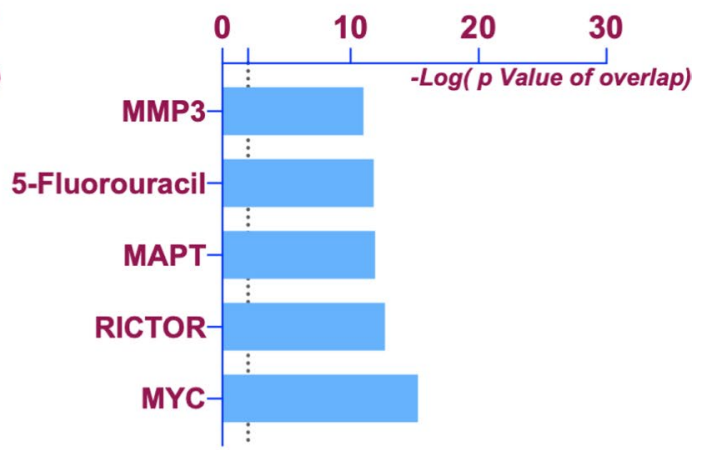

D

E4E4 - AD/C

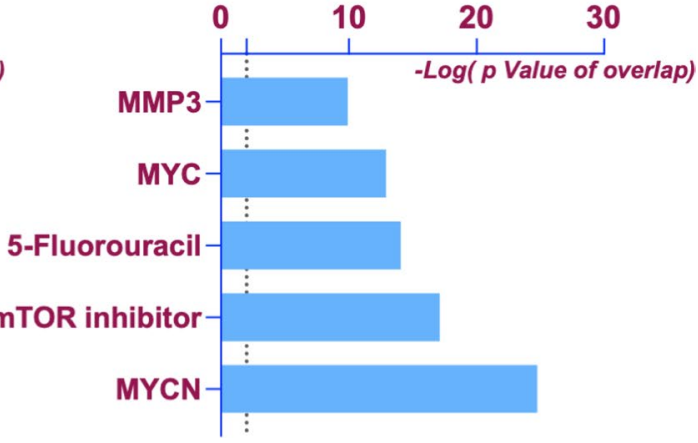

Fig. 8 Upstream regulators implicated in the proteomic changes observed in the cerebrovasculature of the inferior frontal gyrus between Alzheimer's disease (AD) patients and controls from different APOE genotype backgrounds. Histograms shows Top 5 significantly altered upstream regulators identified by ingenuity pathway analyses between AD vs controls from APOE2/E3 (A), APOE3/E3 (B), APOE3/E4 (C) and APOE4/E4 (D) genotypes. Data represents negative Log10 of the FDR adjusted "overlap" P value after Fischer's test and Benjamin Hochberg correction. Overlap $P$ values are generated based on the significant overlap between dataset proteins/genes and known targets regulated by a transcription factor/ upstream regulator. Cut of level for significantly altered upstream regulators was set at $\geq 2$ (i.e. $-\log 10[P$ value])

aspects of energy bioenergetics and immune cell trafficking in the aged brain. These proteomic changes appeared to involve a variety of different cell types, as expression levels of some significant proteins were found to be specific to smooth muscle cells, pericytes, astrocytes and endothelial cells.

Despite the relatively young age of the APOE4/4 cases, a host of molecular abnormalities were observed in their cerebrovessels when compared to the older E3/ E3 cases. Firstly, 260 significant proteins were identified from this comparison, and 25 modulated pathways, most notably EIF2, sirtuin, eIF4/70S6K and mTOR signaling, phagosome maturation, remodeling of adherent junction and DNA repair mechanisms. Of the 25 pathways identified, two were unique to only the E4/E4 vs E3/ E3 group (i.e. Granzyme A and semaphorin signaling). With respect to the cerebrovascular cells playing a role in these changes, we saw significant changes in cell specific markers related to endothelial cells and astrocytes.

Due to the age disparity in the E4/4 vs E3/3 groups and the possibility that the isoform specific effects we observed may be driven in part by differences in age, we also compared differences between E3/4 vs E3/3 groups, which were more closely matched with age. We still 
Table 4 List of Top 25 proteins significantly regulated in the cerebrovasculature of the inferior frontal gyrus in Alzheimer's disease vs controls from APOE3/4 genotype

\begin{tabular}{|c|c|c|c|c|c|}
\hline Gene ID & Label & Protein name & Biological function & $\log 2 \mathrm{FC}$ & $P$ value \\
\hline AINX & Q16352 & $\begin{array}{l}\text { Alpha-internexin (Alpha-Inx) (66 kDa neurofila- } \\
\text { ment protein) }\end{array}$ & Structural constituent of cytoskeleton & 0.845 & $3.38 \mathrm{E}-05$ \\
\hline MYH9 & P35579 & $\begin{array}{l}\text { Myosin-9 (Cellular myosin heavy chain, type A) } \\
\text { (Myosin heavy chain 9) }\end{array}$ & Actin binding & -0.593 & $5.01 \mathrm{E}-05$ \\
\hline Q59GY2 & Q9HBB3 & $60 S$ ribosomal protein $L 6$ & Structural constituent of ribosome & -0.569 & $6.24 \mathrm{E}-05$ \\
\hline B2R8Z8 & Q59GY2 & Ribosomal protein L4 variant (Fragment) & RNA binding & -0.558 & $1.23 \mathrm{E}-04$ \\
\hline A0A024R333 & B5BUB1 & RuvB-like helicase (EC 3.6.4.12) (Fragment) & Chromatin binding & -0.438 & $1.26 \mathrm{E}-04$ \\
\hline ERP29 & B2R8Z8 & $\begin{array}{l}\text { CDNA, FLJ94136, synaptotagmin binding, cyto- } \\
\text { plasmic RNA interacting protein (SYNCRIP) }\end{array}$ & Chaperone binding & -0.663 & $2.19 \mathrm{E}-04$ \\
\hline RS2 & A0A024R333 & Transmembrane protein 113 , isoform CRA_a & Cadherin binding & -0.344 & $4.49 \mathrm{E}-04$ \\
\hline Q6FGH9 & P30040 & $\begin{array}{l}\text { Endoplasmic reticulum resident protein } 29 \\
\text { (ERp29) (Endoplasmic reticulum resident } \\
\text { protein 28) }\end{array}$ & Dynein intermediate chain binding & -0.985 & 5.07E-04 \\
\hline RS16 & P15880 & $\begin{array}{l}40 \text { S ribosomal protein S2 (40S ribosomal protein } \\
\text { S4) }\end{array}$ & RNA binding & -0.662 & $5.51 \mathrm{E}-04$ \\
\hline SRSF7 & Q6FGH9 & Dynein light chain & Protein domain specific binding & -0.465 & $5.64 \mathrm{E}-04$ \\
\hline AOA0S2Z3L2 & P62249 & $\begin{array}{l}40 \text { S ribosomal protein S16 (Small ribosomal } \\
\text { subunit protein uS9) }\end{array}$ & ATP binding & -0.611 & $6.75 \mathrm{E}-04$ \\
\hline Q53F64 & Q16629 & $\begin{array}{l}\text { Serine/arginine-rich splicing factor } 7 \text { (Splicing } \\
\text { factor 9G8) (Splicing factor, arginine/serine- } \\
\text { rich 7) }\end{array}$ & RNA binding & -0.742 & $7.52 E-04$ \\
\hline V9HWK4 & AOA0S2Z3L2 & $\begin{array}{l}\text { ATPase Ca++ transporting cardiac muscle slow } \\
\text { twitch } 2 \text { isoform } 1\end{array}$ & Double-stranded RNA binding & -0.331 & $1.02 \mathrm{E}-03$ \\
\hline A8KAP3 & Q14498 & $\begin{array}{l}\text { RNA-binding protein } 39 \text { (CAPER alpha) (Hepato- } \\
\text { cellular carcinoma protein 1) }\end{array}$ & GTPase activity & -0.396 & 1.03E-03 \\
\hline A8K329 & A0A024R1Q8 & Ribosomal protein L23, isoform CRA_b & RNA binding & -0.488 & $1.09 \mathrm{E}-03$ \\
\hline NFL & Q53F64 & $\begin{array}{l}\text { Heterogeneous nuclear ribonucleoprotein } A B \\
\text { isoform a variant (Fragment) }\end{array}$ & Structural constituent of cytoskeleton & -0.686 & 1.10E-03 \\
\hline CAVN1 & V9HWK4 & Epididymis luminal protein 162 & Identical protein binding & -0.547 & 1.11E-03 \\
\hline J3KTL2 & Q13435 & Splicing factor 3B subunit 2 & Protein kinase $\mathrm{B}$ binding & -0.644 & $1.23 \mathrm{E}-03$ \\
\hline ACTN4 & A8KAP3 & $\begin{array}{l}\text { cDNA FLJ78483, elongation factor Tu GTP bind- } \\
\text { ing domain containing } 2 \text { (EFTUD2) }\end{array}$ & Actin filament binding & -0.429 & $1.66 \mathrm{E}-03$ \\
\hline RL24 & A8K329 & $\begin{array}{l}\text { CDNA FLJ76656, scaffold attachment factor B } \\
\text { (SAFB) }\end{array}$ & Cadherin binding & -0.662 & $1.67 \mathrm{E}-03$ \\
\hline OGT1 & P07196 & $\begin{array}{l}\text { Neurofilament light polypeptide (NF-L) (68 kDa } \\
\text { neurofilament protein) }\end{array}$ & $\begin{array}{l}\text { Phosphatidylinositol-3,4,5-trisphosphate bind- } \\
\text { ing }\end{array}$ & 1.164 & $1.86 \mathrm{E}-03$ \\
\hline B1PS43 & Q6NZI2 & Caveolae-associated protein 1 (Cavin-1) & Actin filament binding & -0.463 & $1.94 \mathrm{E}-03$ \\
\hline RL3 & J3KTL2 & Serine/arginine-rich-splicing factor 1 & Structural constituent of ribosome & -0.711 & $1.98 \mathrm{E}-03$ \\
\hline B4DEG4 & O43707 & Alpha-actinin-4 (Non-muscle alpha-actinin 4) & RNA binding & -0.495 & $2.02 \mathrm{E}-03$ \\
\hline PLEC & AOAOA6YYL6 & Protein RPL17-C18orf32 & Structural constituent of cytoskeleton & -0.565 & $2.23 \mathrm{E}-03$ \\
\hline
\end{tabular}

Tables show the biological functions of the top 25 significant proteins between AD vs control from the APOE3/E4 genotype

observed fifty nine significant proteins from this comparison, and seven modulated pathways, the notable ones being EIF2 signaling, TCA cycle, Calcium transport, HIF $\alpha$ and GM-CSF signaling. Given that EIF2 signaling was the top significantly modulated pathway between E4/ E4 vs E3/E3 and E3/E4 vs E3/E3 cases, it suggests that APOE4 genotype may play a prominent role in regulating this molecular signaling pathway essential for protein translation.
Together, our findings reveal that underlying APOE genotype-specific proteomic differences in nondemented controls exists and may explain the early changes to cerebrovessels and the subsequent propensity for developing AD particularly in APOE4 carriers. On the other hand, the E2 specific effects we observed could also explain the association of this allele with an increased risk for CAA in the elderly, development of cerebral microhemorrhages in CAA patients, and an increased 
Table 5 List of Top 25 proteins significantly regulated in the cerebrovasculature of the inferior frontal gyrus in Alzheimer's disease vs controls from APOE4/4 genotypes

\begin{tabular}{|c|c|c|c|c|c|}
\hline Gene ID & Label & Protein name & Biological function & $\log 2 \mathrm{FC}$ & $P$ value \\
\hline RBM39 & Q14498 & $\begin{array}{l}\text { RNA-binding protein } 39 \text { (CAPER alpha) (Hepatocel- } \\
\text { lular carcinoma protein 1) }\end{array}$ & RNA binding & 0.594 & 0.000 \\
\hline Q59F66 & Q59F66 & $\begin{array}{l}\text { DEAD box polypeptide } 17 \text { isoform p82 variant (Frag- } \\
\text { ment) }\end{array}$ & Nucleic acid binding & 0.476 & 0.001 \\
\hline XRCC5 & P13010 & X-ray repair cross-complementing protein 5 & Damaged DNA binding & 0.752 & 0.001 \\
\hline PSD11 & 000231 & $\begin{array}{l}\text { 26S proteasome non-ATPase regulatory subunit } 11 \\
\text { (26S proteasome regulatory subunit RPN6) }\end{array}$ & Structural molecule activity & 0.899 & 0.001 \\
\hline A0A024RAZ7 & A0A024RAZ7 & $\begin{array}{l}\text { Heterogeneous nuclear ribonucleoprotein A1, } \\
\text { isoform CRA_b }\end{array}$ & RNA binding & 0.694 & 0.001 \\
\hline DX39B & Q13838 & Spliceosome RNA helicase DDX39B & ATPase activity & 0.564 & 0.001 \\
\hline E7EMK3 & E7EMK3 & Flotillin-2 & Protease binding & -0.731 & 0.001 \\
\hline B2R673 & B2R673 & $\begin{array}{l}\text { Dihydrolipoamide acetyltransferase component of } \\
\text { pyruvate dehydrogenase complex }\end{array}$ & Transferase activity, transferring acyl groups & -0.605 & 0.001 \\
\hline SART3 & Q15020 & $\begin{array}{l}\text { Squamous cell carcinoma antigen recognized by } \\
\text { T-cells } 3 \text { (SART-3) }\end{array}$ & Histone binding & 0.571 & 0.001 \\
\hline ENOA & P06733 & Alpha-enolase & Cadherin binding & -0.512 & 0.001 \\
\hline Q9HBB3 & Q9HBB3 & $60 S$ ribosomal protein $L 6$ & DNA binding & 0.227 & 0.002 \\
\hline PUF60 & Q9UHX1 & $\begin{array}{l}\text { Poly(U)-binding-splicing factor PUF60 (60 kDa } \\
\text { poly(U)-binding-splicing factor) }\end{array}$ & Cadherin binding & 0.612 & 0.002 \\
\hline Q53SS8 & Q53SS8 & $\begin{array}{l}\text { Epididymis secretory protein Li } 85 \text { (Poly(RC) binding } \\
\text { protein 1) }\end{array}$ & DNA-binding transcription factor activity & 0.524 & 0.002 \\
\hline AOA0A6YYL6 & A0A0A6YYL6 & Protein RPL17-C18orf32 & Structural constituent of ribosome & 0.364 & 0.002 \\
\hline Q6ICQ8 & Q6ICQ8 & $\begin{array}{l}\text { ARHG protein (Ras homolog gene family, member G } \\
(\text { Rho G)) }\end{array}$ & GTPase activity & -0.500 & 0.002 \\
\hline B5BU24 & B5BU24 & 14-3-3 protein beta/alpha & & -0.462 & 0.003 \\
\hline SF3B2 & Q13435 & Splicing factor 3 B subunit 2 & RNA binding & 0.435 & 0.003 \\
\hline A0A0C4DG89 & A0A0C4DG89 & Probable ATP-dependent RNA helicase DDX46 & ATP binding & 0.595 & 0.003 \\
\hline U520 & O75643 & U5 small nuclear ribonucleoprotein 200 kDa helicase & Helicase activity and ATP binding & 0.420 & 0.003 \\
\hline B2R7W4 & B2R7W4 & $\begin{array}{l}\text { CDNA, FLJ93632, heterogeneous nuclear ribonucleo- } \\
\text { protein R (HNRPR), mRNA }\end{array}$ & RNA binding & 0.675 & 0.003 \\
\hline A0A0S2Z4Z9 & A0A0S2Z4Z9 & $\begin{array}{l}\text { Non-POU domain containing octamer-binding } \\
\text { isoform } 1 \text { (Fragment) }\end{array}$ & RNA binding & 0.588 & 0.003 \\
\hline KPCB & P05771 & Protein kinase $C$ beta type (PKC-B) (PKC-beta) & Calcium channel regulator activity & -0.233 & 0.004 \\
\hline B5BUB1 & B5BUB1 & RuvB-like helicase (EC 3.6.4.12) (Fragment) & 5'-3'DNA helicase activity & 0.258 & 0.004 \\
\hline B5BUB5 & B5BUB5 & Autoantigen La (Fragment) & RNA binding & 1.177 & 0.004 \\
\hline PARP1 & P09874 & Poly [ADP-ribose] polymerase 1 (PARP-1) & DNA binding & 0.625 & 0.004 \\
\hline
\end{tabular}

Tables show the biological functions of the top 25 significant proteins between AD vs controls cases from the APOE4/E4 genotype background

likelihood for cerebral ischemia in the elderly (independent of AD/amyloid pathology) [64-67]. Notably, the E2 genotype is also commonly attributed to hyperlipidemia and cardiovascular issues in the general population [68, 69]. Paradoxically, APOE 2 also reduces the risk for CAA in $\mathrm{AD}$ patients, and protects against AD-related pathology compared to other APOE variants, as evidenced by the low frequency of E2/E2 AD cases in the general population [70,71]. How these underlying cerebrovascular changes influences AD pathobiology remains elusive. Perhaps a limitation of this study is that we have not captured the extravascular related effects of E2 which may explain its protective role in mitigating $\mathrm{AD}$ pathogenesis. Further studies need to be explored in this genotype to identify the specific mechanisms driving their reduced risk for developing AD.

\section{APOE specific effects on cerebrovascular proteome in AD}

We next interrogated the interaction between APOE genotype and disease (i.e. AD diagnosis) from our cerebrovascular proteomic analyses. The demographics of the $\mathrm{AD}$ patients we used in this study revealed that all genotypes consisted of mixed gender, and all cases were octogenarians with a mean age between 84 and 89 yrs, except 
the E3/E4 cases which were the youngest with a mean age of 78yrs. As expected, the E4/E4 cases showed the most aggressive clinicopathological scores, with the worst scores for Tau, amyloid, CAA and white matter pathology, and the smallest brain weight on average compared to all other genotypes, while the E2/3 cases on the other hand demonstrated the least aggressive clinicopathological scores.

Our proteomic analyses of E4 carriers revealed 192 and 189 significantly regulated proteins that were unique to the interaction between disease and genotype, E3/E4 or E4/E4 respectively. Ingenuity pathway analyses identified, 24 and 14 pathways between disease and genotype interaction, E3/E4 or E4/E4 respectively. EIF2, eIF4 and $70 \mathrm{~S} 6 \mathrm{~K}$, and mTOR signaling were amongst the top significantly altered pathways from the disease and APOE (E3/E4 or E4/E4) genotype interactions, signifying that the APOE4 allele influences the molecular mechanisms driving protein translation/synthesis, metabolic pathways, and UPR/ER stress and autophagy. Because we observed similar biological functions altered between the E4/E4 vs E3/E3 controls, it is likely E4 genotype drives early cerebrovascular abnormalities in the aging process, which increases the vulnerability of the cerebrovasculature to further damage in $\mathrm{AD}$ pathogenesis. To corroborate our findings, previous studies have suggested that EIF $2 \alpha$ and mTOR pathways are major players in the APOE4 mediated cellular effects, and have been explored as therapeutic targets in mouse models of AD [72-76].

Of the total number of significantly altered pathways identified, 6 out of 14 were unique to only the disease and E4/E4 interactions, while 17 out of 24 were unique to the disease and E3/E4 interaction. Some of the Top pathways unique to the E4/E4 groups include Tight junction signaling, neucleobases (pyramidine) synthesis, and Integrin linked kinase signaling, and from the E3/E4 groups, sirtuin signaling pathway, remodeling of adherene junctions and Telemore extension.

With respect to the cerebrovascular cells playing a role in these changes, we saw significant changes in protein markers specific to smooth muscle cells, endothelial cells and astrocytes with disease and (E3/ E4) genotype interaction, while only endothelial cells and astrocyte specific markers were identified from the E4/E4 groups.

Together our findings implicate a specific prominent role for the APOE4 allele in molecular mechanisms such as protein translation/synthesis, cell to cell contact signaling, BBB integrity, nucleic acid synthesis, cellular survival and metabolism, and ER stress/autophagy in cerebrovascular cells. Given that the E4 carriers showed the worst clinicopathological phenotypes that positively correlated with the extent of cerebrovascular abnormalities, we consider that these molecular mechanisms identified herein may provide tractable cerebrovascular specific targets for disease modifying strategies in $\mathrm{AD}$, particularly for subjects carrying the high risk APOE4 genotype.

In contrast, our proteomic analyses of E3/E3 and E2/ E3 carriers revealed 41 and 102 significantly regulated proteins that were unique to the interaction between disease and these respective APOE genotype interactions. Ingenuity pathway analyses identified 16 pathways from the disease and E3/E3 interaction and 5 pathways from the disease and E2/E3 interaction. Noted pathways modulated in the E3/E3 cohort include EIF2, mTOR, eIF4 and p70S6K signaling, phagosome maturation, glutamine synthesis, CXCR4 signaling and aldosterone signaling. While Spliceosomal cycle, DNA methylation and transcriptional repression signaling, EIF2 signaling, DNA base excision repair pathway and DNA double strand break repair signaling were the only five pathways altered in the E2/E3 cohort.

Eleven out of 16 pathways were unique to the disease and E3/E3 genotype interaction, while 1 out of 5 was unique to the disease and E2/E3 genotype interaction. The top two pathways unique to the E3/E3 cohorts were CXCR4 and aldosterone signaling, while DNA methylation and transcriptional repression signaling was the only pathway unique to E2/E3.

With respect to the cerebrovascular cells potentially influencing these proteomic changes, we saw significant changes in cell markers specific to only astrocytes from E2/E3 and E3/E3 AD cases vs controls, suggesting that E2 and E3 allele may play less prominent roles on endothelial and mural cell pathobiology in AD compared to the E4 allele. Despite the unfavorable cerebrovascular changes observed in non-demented E2 allele carriers, E2/3 AD cases did not exhibit an augmented cerebrovascular phenotype in $\mathrm{AD}$ compared to the other $\mathrm{APOE}$ variants; their unique $\mathrm{AD}$-related phenotype was typified by alterations in nucleic acid synthesis, transcriptional gene pathways and protein translation. Given that the molecular events in cerebrovessels differ significantly in the APOE2 genotype compared to other variants, targeting these distinct pathways in $\mathrm{AD}$, may provide a unique strategy for combating the specific cerebrovascular changes in those carrying this alleles.

To our knowledge no studies have directly investigated the influence of APOE genotype on proteomic changes in cerebrovascular tissue from AD brains. The most relevant study we found used a comparative unbiased mass spectrometry-based method to interrogate post-mortem brain cortical tissues from pathologically-defined AD cases from E2/E3, E3/E3 and E4/E4 genotypes compared to control E3/E3 cases [77]. Using a weighted co-expression network analysis method, the authors identified 33 
modules of co-expressed proteins, 12 of which were significantly different by APOE genotype in AD. The top modules impacted by E4/E4 genotype included mitochondrial function, synaptic transmission, inflammatory response, and a trend for protein translation, corroborating some of our findings in the E3/E4 and E4/E4 genotypes. Unlike our findings, their deconvolution cell type analyses showed a lack of effect on specific phenotypic cell type markers observed in the AD cases on the E3/ E3 and E4/E4 background when compared to control E3/ E3 cases. This was possibly due to the lack of appropriate controls for each genotype in the study design. The authors, however, did note that E2 allele carriers suppressed homeostatic and disease-associated AD phenotypic cell type marker changes in astrocytes, microglia, and endothelia, pointing to a possible neuroprotective feature of the E2 allele [77].

The precise mechanisms driving APOE genotype specific effects on neurological dysfunction has been largely discussed in the literature. Some of the earliest clues linking APOE with vascular degeneration in AD involved studies demonstrating a link between APOE- $\varepsilon 4$ and increased amyloid deposition around blood vessels [38, $78-80]$. APOE is thought to play a role in regulating the metabolism and perivascular drainage of $A \beta$ from extracellular fluids in the brain. Intracerebral injection of $A \beta$ into the brain of mice results in co-localization with APOE along basement membrane drainage pathways in the walls of cerebral arteries [81]. This clearance route is likely deficient in APOE4 carriers, as shown by the colocalization of APOE and non-fibrillar A $\beta$ in the perivascular space and neuropil surrounding cerebral arteries of human AD brains [82] and also preclinical transgenic models of amyloidosis on the human APOE background [81, 83-86]. This event is possibly driven by APOE early in the sequelae of $\mathrm{AD}$, as APOE has been found to accumulate in the early stages of senile plaque formation, preceding $A \beta$ deposition in meningeal vessels in amyloid angiopathy [87]. Further support of this role on amyloid clearance, have also been corroborated by studies showing that APOE $4 / \mathrm{A} \beta$ complexes form a weaker bond formation with basement membrane proteins along cerebral arteries compared to APOE3/A $\beta$ complexes [86]. Other studies have also shown a direct impact of APOE on BBB permeability by altering transport mechanisms at the level of endothelial cells $[88,89]$. For example, studies in APOE knock-in mice have revealed an age-related (12Mos $>6 \mathrm{Mos})$ and isoform specific (E4 $>$ E3) increase in the mRNA levels of influx and efflux $A \beta$ transporters [89]. We also previously showed that APOE4 isoform can influence MMP9 induced shedding of an efflux transporter (LRP-1) of $A \beta$ and its subsequent BBB transport of APOE-A $\beta$ complexes [59]. The consequences of
$\mathrm{APOE}$ on $\mathrm{A} \beta$ clearance and the subsequent increase in toxic amyloid deposits along the walls of the vasculature could be a plausible mechanism driving the toxicity of cerebrovessels in the pathogenesis of $\mathrm{AD}$, leading to the molecular abnormalities we observed in this study.

Other studies have provided an alternate narrative of these events that implicates a direct role for APOE on cerebrovessel damage independent of its effects on $A \beta$. Early clues to support this hypothesis originated from seminal work showing a significant correlation between APOE allele and cerebral small-vascular diseases (CSVD) typified by white matter hyperintensities, dilated perivascular space, microbleeds and lacunae $[40,41,67]$. Severity of CSVD has been reported to correlate with the localization of APOE and plasma derived IgG proteins around CSVD altered cerebrovessel walls [40, 41]. Studies have also shown an association between APOE4 and atherosclerotic middle cerebral artery stenosis in cerebral ischemia [43], and a relation between coronary atherosclerosis and APOE4 genotypes in AD cases [44]. These effects have been suggested to be influenced in part by the impact of APOE4 allele on peripheral lipid profiles (i.e. higher total cholesterol, LDL cholesterol and apoliporotein B levels in the plasma) [69] and the innate immune response, and their combined indirect influence on cerebrovessels.

Other investigators have speculated that changes in APOE levels in the brain may drive AD pathogenesis [90]. We therefore interrogated the levels of APOE in cerebrovessels of control and $\mathrm{AD}$ cases, and observed no significant change across genotype in non-demented cases, but unexpectedly saw a significant increase in E4/ E4 AD cases compared to controls. Although some studies have reported an inverse correlation between brain APOE expression and $A \beta$ load $[90,91]$, a closer look at the literature suggests inconsistent results when measuring APOE levels in the brain as a function of APOE genotype [92-98]. In our study, the E4/E4 AD and control cases were poorly age-matched, which could be a driving factor in the differences observed. Larger cohorts of agematched cases will be needed to confirm this finding in E4 carriers, and to demonstrate whether this is a unique feature observed in the cerebrovessels of E4 carriers. Further studies will also be needed to investigate the cellular origin and upstream regulators driving these changes, how APOE expression levels impacts on cerebrovascular cell dysfunction, and whether this event occurs prior to the onset of $\mathrm{A} \beta$ deposition.

More recent work has confirmed the link between APOE4 and the accelerated breakdown of the BBB in vulnerable brain regions such as the medial temporal lobe in unimpaired, and to a greater extent in cognitively impaired patients, independent of amyloid and tau 
pathology $[46,47,49]$. This has also been confirmed in human APOE4 expressing mice showing early vascular changes (such as BBB damage, influx of neurotoxic serum proteins, early microvascular and cerebral blood flow reductions [42]) that precede the early onset of neuronal dysfunction and subsequent neurodegeneration. This effect is thought to be mediated by the influence of APOE on pericyte dysfunction. Early increase in CSF levels of soluble PDGFR $\beta$, degeneration of pericytes, and their coverage of brain capillaries have been shown in human and transgenic AD models on the APOE4 background $[42,47,99,100]$. Damage to pericytes could impact on the vasoconstrictive properties of cerebral microvessels leading to diminished blood flow, impaired perivascular drainage, and cognitive decline [101-103]. These effects on BBB integrity and pericyte dysfunction have been shown to be mediated by the increase in Cyclophilin (Cyp) production, which activates NF-kB induced upregulation of MM9, involved in degrading capillary basement membrane and tight junction proteins [42, 47, 99]. Consistent with our study, damage to BBB integrity, aberrant regulation of protein translation, deficits in cellular survival and metabolism, and autophagy were notably altered in APOE4 cases with AD. Interfering with these pathways and those identified in our studies, using pharmacological inhibitors, short interfering RNA, or genetic deletion may provide an avenue to reverse weakened cerebrovascular integrity and BBB dysfunction in AD.

Another possible explanation of the influence of APOE on cerebrovascular dysfunction could be through its indirect effect on inflammatory processes in glial cells. Previous work has shown that glial cells play an essential role in driving APOE4 mediated neurodegeneration in preclinical models of AD-like pathology [104-107]. APOE4 has also been shown to drive widespread molecular and cellular alterations associated with AD pathogenesis in isogenic APOE carrying glial cells derived from inducible pluripotent stem cells (iPSC) from AD patients [108]. Our cerebrovascular tissue showed moderate expression of glial markers, therefore some of the APOE specific effects we observed could be driving in part by changes in these cell types, which are the main producers of APOE in the brain [33]. APOE4 genotype has been demonstrated to influence the phenotypic change of astrocytes into the A1 disease phenotype, and also convert microglia into neurotoxic entities that produce proinflammatory cytokines [105, 108-112]. In AD, glial cell inflammation could therefore serve as critical determinants driving the immune response that contributes to the damage of cerebrovessels. In this study, we saw several astrocyte cell specific markers (e.g. APOE, GFAP, S100B, $A Q 4)$ that were significantly altered in control vs $A D$ cases from all APOE backgrounds. This correlated with changes in serum derived proteins such as thrombin factors, increased expression of integrins, adhesion molecules and chemokines between $\mathrm{AD}$ cases and controls, in E3 and E4 carriers. Increased expression of adhesion molecules and integrins can make vessels more adhesive, whereby immune cells such as macrophages/foamy cells and neutrophils could infiltrate the brain environment, and also clog up vessel walls leading to a reduction in blood flow and inflammatory damage to cerebrovessels [113].

\section{Conclusion}

Our work has provided a snapshot of the molecular changes that exists in the cerebrovasculature of $A D$ and non-demented brains from different APOE genotypes. One limitation of this study design is our inability to determine whether these disrupted cellular functions precede or are a direct consequence of amyloid or tau pathology in AD. A larger sample size (well matched for all genotypes, sex and age) and longitudinal study design focusing on the neuropathological staging of disease will be needed to elucidate the specific effect of APOE on the sequelae of cerebrovascular dysfunction in AD pathobiology. Future studies could also incorporate phosphoproteomic approaches to identify phosphorylation status of EIF2 and mTOR specific pathways that were highly enriched in our proteomic datasets. The use of single cell approaches with tools such as FACS, single cell transcriptomics (scRNAseq) and RNAscope may also be invaluable, and will complement our total proteomic method which lacked the ability to detect the specific cellular origins of each differentially expressed proteins.

Together, our findings in this study have provided novel insights into the impact of APOE genotype on the brain vasculature in normal and $\mathrm{AD}$ conditions. The cerebrovasculature is crucial in maintaining brain homeostasis, neurovascular coupling, and supplying oxygen and glucose to the highly active cells of the brain. We consider that the APOE dependent changes identified herein may generate novel targets for developing therapies that protect against damage to the cerebrovasculature and mitigate the risk for AD conferred by APOE genotype. 


\section{Supplementary Information}

The online version contains supplementary material available at https://doi. org/10.1186/s13041-021-00803-9.

Additional file 1: Table S1. List of significantly regulated proteins in the cerebrovasculature of the inferior frontal gyrus in healthy homozygote control cases from APOE2/E2 vs APOE3/E3 genotypes. Data are expressed as the negative $\log 10$ of the $p$ value (green horizontal bars - significance cut off set at $>1.3$ ), and the Log2 fold change between control cases from APOE2/E2 vs APOE3/E3 genotypes. Heat map indicates downregulated (Red box) or upregulated (Blue box) proteins. Statistical analyses was performed using two way ANOVA after logarithmic transformation.

Additional file 2: Table S2.List of significantly regulated proteins in the cerebrovasculature of the inferior frontal gyrus in healthy homozygote control cases from APOE4/E4 vs APOE3/E3 genotypes. Data are expressed as the negative $\log 10$ of the $p$ value (green horizontal bars-significance cut off set at >1.3), and the Log2 fold change between control cases from APOE4/E4 vs APOE3/E3 genotypes Heat map indicates downregulated (Red box) or upregulated (Blue box) proteins. Statistical analyses was performed using two way ANOVA after logarithmic transformation.

Additional file 3: Table S3. List of significantly regulated proteins in the cerebrovasculature of the inferior frontal gyrus in healthy homozygote control cases from APOE3/E4 vs APOE3/E3 genotypes. Data are expressed as the negative Log10 of the $p$ value (green horizontal bars-significance cut off set at $>1.3$ ), and the Log2 fold change between control cases from APOE3/E4 vs APOE3/E3 genotypes Heat map indicates downregulated (Red box) or upregulated (Blue box) proteins. Statistical analyses was performed using two way ANOVA after logarithmic transformation.

Additional file 4: Table S4. List of significantly regulated proteins in the cerebrovasculature of the inferior frontal gyrus in Alzheimer's disease and matched control cases from E2/E3 genotypes. Data are expressed as the negative Log 10 of the $p$ value (green horizontal bars - significance cut off set at > 1.3), and the Log2 fold change between AD and matched control cases from E2/E3 genotypes. Heat map indicates downregulated (Red box) or upregulated (Blue box) proteins. Statistical analyses was performed using two way ANOVA after logarithmic transformation.

Additional file 5: Table $\mathbf{S 5}$. List of significantly regulated proteins in the cerebrovasculature of the inferior frontal gyrus in Alzheimer's disease and matched control cases from E3/E3 genotypes. Data are expressed as the negative $\log 10$ of the $p$ value (green horizontal bars - significance cut off set at > 1.3), and the Log2 fold change between AD and matched control cases from E3/E3 genotypes. Heat map indicates downregulated (Red box) or upregulated (Blue box) proteins. Statistical analyses was performed using two way ANOVA after logarithmic transformation.

Additional file 6: Table S6. List of significantly regulated proteins in the cerebrovasculature of the inferior frontal gyrus in Alzheimer's disease and matched control cases from E3/E4 genotypes. Data are expressed as the negative Log 10 of the $p$ value (green horizontal bars-significance cut off set at > 1.3), and the Log2 fold change between AD and matched control cases from E3/E4 genotypes. Heat map indicates downregulated (Red box) or upregulated (Blue box) proteins. Statistical analyses was performed using two way ANOVA after logarithmic transformation.

Additional file 7: Table S7. List of significantly regulated proteins in the cerebrovasculature of the inferior frontal gyrus in Alzheimer's disease and matched control cases from E4/E4 genotypes. Data are expressed as the negative Log 10 of the $p$ value (green horizontal bars - significance cut off set at > 1.3), and the Log2 fold change between AD and matched control cases from E4/E4 genotypes. Heat map indicates downregulated (Red box) or upregulated (Blue box) proteins. Statistical analyses was performed using two way ANOVA after logarithmic transformation.

\section{Acknowledgements}

We are grateful to the Banner Sun Health Research Institute Brain and Body Donation Program of Sun City, Arizona for providing the human brain specimens. The Brain and Body Donation Program is supported by the National Institute of Neurological Disorders and Stroke (U24 NS072026 National Brain and Tissue Resource for Parkinson's Disease and Related Disorders), the National Institute on Aging (P30 AG19610 Arizona Alzheimer's Disease Core Center), the Arizona Department of Health Services (contract 211002, Arizona Alzheimer's Research Center), the Arizona Biomedical Research Commission (contracts 4001, 0011, 05-901 and 1001 to the Arizona Parkinson's Disease Consortium) and the Michael J. Fox Foundation for Parkinson's Research. Human brain tissue specimens were also obtained from the University of Maryland Brain and Tissue Bank which is a Brain and Tissue Repository of the $\mathrm{NIH}$ NeuroBioBank (Baltimore, MD) and the Mount Sinai NIH Brain and Tissue Repository (New York, NY). Finally, we extend our appreciation to the Roskamp Institute for their generosity in helping to make this work possible.

\section{Disclosures}

Dr. Bachmeier is a Research Scientist at the Bay Pines VA Healthcare System, Bay Pines, FL. Dr. Crawford is a Research Career Scientist at the James A. Haley Veterans Hospital, Tampa, FL. The content is solely the responsibility of the authors and does not necessarily represent the official views of the National Institutes of Health, the Department of Veterans Affairs, or the United States Government.

\section{Authors' contributions}

$\mathrm{CB}$ and JO conceived and directed the project. JO, GC, JMR, JE analyzed the results. JO, PV, BS, ME, AE carried out the experiments. JO, CB and FC drafted the manuscript. FC, MM were consultants on the project and provided their expertise and interpretation of the data. All authors read and approved the final manuscript.

\section{Funding}

This work was supported by the National Institute on Aging of the National Institutes of Health under award number R01AG041971. The funding body did not have any role in the design of the study, the analysis and interpretation of data, nor in the preparation of the manuscript.

\section{Availability of data and materials}

Our mass spectrometry data has been deposited into the ProteomeXchange Consortium via the PRIDE partner repository. Our datasets can be located with the unique identifier - PXD023340

\section{Declarations}

\section{Ethics approval and consent to participate}

All research in this study was performed in accordance with the United States (US) National Institutions of Health guidelines for research involving human tissues. Brain tissue were obtained from Dr. Thomas Beach, Director of the Brain and Body Donation Program at Sun Health Research Institute (Sun City, AZ), and the NIH NeuroBioBank at the Ican School of Medicine at Mount Sinai and University of Maryland, Baltimore. Approval and permission to use human brain tissue for research was obtained from the NIH NeuroBioBank in accordance with the institutional bioethics guidelines. All donors and their families gave written informed consent for autopsy and use of brain tissue for research purposes. As samples were obtained from deceased, de-identified, consenting individuals, no further ethical approval was required.

\section{Consent for publication}

Not applicable as brain samples were collected post-mortem and de-identified.

\section{Competing interests}

The authors declare that they have no competing interests.

\section{Author details}

${ }^{1}$ Department of Experimental Neuropathology, Roskamp Institute, Sarasota, FL 34243, USA. ${ }^{2}$ James A. Haley Veterans' Hospital, Tampa, FL, USA. ${ }^{3}$ The Open University, Milton Keynes, UK. ${ }^{4}$ Boehringer Ingelheim Pharmaceuticals, Inc., Ridgefield, CT, USA. ${ }^{5}$ Bay Pines VA Healthcare System, Bay Pines, FL, USA.

Received: 27 January 2021 Accepted: 4 June 2021

Published online: 08 July 2021 


\section{References}

1. de la Torre J. The vascular hypothesis of Alzheimer's Disease: a key to preclinical prediction of dementia using neuroimaging. Mecocci P, editor. J Alzheimers Dis. 2018;63:35-52. https://doi.org/10.3233/ JAD-180004

2. De la Torre JC, Mussivand T. Can disturbed brain microcirculation cause Alzheimer's disease? Neurol Res. 1993;15:146-53. https://doi.org/10. 1080/01616412.1993.11740127.

3. Di Marco LY, Venneri A, Farkas E, Evans PC, Marzo A, Frangi AF. Vascular dysfunction in the pathogenesis of Alzheimer's disease-a review of endothelium-mediated mechanisms and ensuing vicious circles. Neurobiol Dis. 2015;82:593-606

4. Binnewijzend MAA, Benedictus MR, Kuijer JPA, van der Flier WM, Teunissen CE, Prins ND, et al. Cerebral perfusion in the predementia stages of Alzheimer's disease. Eur Radiol. 2016;26:506-14.

5. Hays CC, Zlatar ZZ, Wierenga CE. The utility of cerebral blood flow as a biomarker of preclinical Alzheimer's disease. Cell Mol Neurobiol. 2016:36:167-79. https://doi.org/10.1007/s10571-015-0261-z.

6. Attems J, Jellinger K, Thal DR, Van Nostrand W. Review: sporadic cerebral amyloid angiopathy. Neuropathol Appl Neurobiol. 2011;37:75-93.

7. Snowdon DA. Aging and Alzheimer's disease: Lessons from the Nun Study. Gerontologist. 1997;37:150-6. https://doi.org/10.1093/geront/ 37.2.150.

8. Snowdon DA. Brain infarction and the clinical expression of Alzheimer disease: the Nun Study. JAMA J Am Med Assoc. 1997;277:813-7. https:// doi.org/10.1001/jama.277.10.813.

9. Nagy Z, Esiri MM, Jobst KA, Morris JH, King EMF, Mcdonald B, et al. The effects of additional pathology on the cognitive deficit in Alzheimer disease. J Neuropathol Exp Neurol. 1997;56:165-70. https://doi.org/10 1097/00005072-199702000-00007.

10. Roher AE, Esh C, Rahman A, Kokjohn TA, Beach TG. Atherosclerosis of cerebral arteries in Alzheimer disease. Stroke. 2004;35:2623-7.

11. Yarchoan M, Xie SX, Kling MA, Toledo JB, Wolk DA, Lee EB, et al. Cerebrovascular atherosclerosis correlates with Alzheimer pathology in neurodegenerative dementias. Brain. 2012:135:3749-56.

12. Beach TG, Wilson JR, Sue LI, Newell A, Poston M, Cisneros R, et al. Circle of Willis atherosclerosis: association with Alzheimer's disease, neuritic plaques and neurofibrillary tangles. Acta Neuropathol. 2007;113:13-21. https://doi.org/10.1007/s00401-006-0136-y.

13. Østergaard L, Aamand R, Gutiérrez-Jiménez E, Ho YCL, Blicher JU, Madsen SM, et al. The capillary dysfunction hypothesis of Alzheimer's disease. Neurobiol Aging. 2013;34:1018-31.

14. Kimura T, Hashimura T, Miyakawa T. Observations of microvessels in the brain with Alzheimer's disease by the scanning electron microscopy. Psychiatry Clin Neurosci. 1991;45:671-6.

15. Hashimura T, Kimura T, Miyakawa T. Morphological changes of blood vessels in the brain with Alzheimer's disease. Psychiatry Clin Neurosci. 1991;45:661-5. https://doi.org/10.1111/j.1440-1819.1991.tb01187.x.

16. Miyakawa T, Kuramoto R. Ultrastructural study of senile plaques and microvessels in the brain with Alzheimer's disease and Down's syndrome. Ann Med. 1989;21:99-102.

17. Farkas E, De Jong Gl, De Vos RAl, Jansen Steur ENH, Luiten PGM. Pathological features of cerebral cortical capillaries are doubled in Alzheimer's disease and Parkinson's disease. Acta Neuropathol. 2000;100:395-402. https://doi.org/10.1007/s004010000195.

18. Deane R, Zlokovic B. Role of the blood-brain barrier in the pathogenesis of Alzheimers disease. Curr Alzheimer Res. 2007:4:191-7.

19. Miyakawa T. Vascular pathology in Alzheimer's disease. Psychogeriatrics. 2010;10:39-44. https://doi.org/10.1111/j.1479-8301.2009.00294.x.

20. Erkinjuntti T, Román G, Gauthier S, Feldman H, Rockwood K. Emerging therapies for vascular dementia and vascular cognitive impairment. Stroke. 2004;35:1010-7. https://doi.org/10.1161/01.STR.0000120731. 88236.33

21. Kalaria RN. Comparison between Alzheimer's disease and vascular dementia: implications for treatment. Neurol Res. 2003;25:661-4. https://doi.org/10.1179/016164103101201968.

22. Custodio N, Montesinos R, Lira D, Herrera-Pérez E, Bardales Y, ValerianoLorenzo L. Demência mista: Revisão das evidências. Dement e Neuropsychol. 2017;11:364-70.
23. Govindpani K, McNamara LG, Smith NR, Vinnakota C, Waldvogel HJ, Faull RL, et al. Vascular dysfunction in Alzheimer's disease: a prelude to the pathological process or a consequence of it? J Clin Med. 2019;8:651.

24. Mosconi L. Brain glucose metabolism in the early and specific diagnosis of Alzheimer's disease: FDG-PET studies in $\mathrm{MCl}$ and AD. Eur J Nucl Med Mol Imaging. 2005;32:486-510. https://doi.org/10.1007/ s00259-005-1762-7.

25. Mawuenyega KG, Sigurdson W, Ovod V, Munsell L, Kasten T, Morris JC, et al. Decreased clearance of CNS $\beta$-amyloid in Alzheimer's disease. Science. 2010;330:1774. https://doi.org/10.1126/science.1197623.

26. Ince PG. Pathological correlates of late-onset dementia in a multicentre, community-based population in England and Wales. Lancet. 2001;357:169-75.

27. Thal DR, Ghebremedhin E, Orantes M, Wiestler OD. Vascular pathology in Alzheimer disease: correlation of cerebral amyloid angiopathy and arteriosclerosis/lipohyalinosis with cognitive decline. J Neuropathol Exp Neurol. 2003;62:1287-301.

28. Tian J, Shi J, Bailey K, Mann DMA. Relationships between arteriosclerosis, cerebral amyloid angiopathy and myelin loss from cerebral cortical white matter in Alzheimer's disease. Neuropathol Appl Neurobiol. 2004;30:46-56.

29. Kalback W, Esh C, Castaño EM, Rahman A, Kokjohn T, Luehrs DC, et al. Atherosclerosis, vascular amyloidosis and brain hypoperfusion in the pathogenesis of sporadic Alzheimer's disease. Neurol Res. 2004;26:525-39.

30. Liu CC, Kanekiyo T, Xu H, Bu G. Apolipoprotein e and Alzheimer disease: risk, mechanisms and therapy. Nat Rev Neurol. 2013;9:106-18.

31. El SS, Costanian C, Kassir R, Visvkis-Siest S, Bissar-Tadmouri N. APOE genotypes in Lebanon: distribution and association with hypercholesterolemia and Alzheimer's disease. Per Med. 2019;16:15-23. https://doi. org/10.2217/pme-2018-0067.

32. Chartier-hariln MC, Parfitt M, Legrain S, Pérez-tur J, Brousseau T, Evans A, et al. Apolipoprotein e, $\varepsilon 4$ allele as a major risk factor for sporadic early and late-onset forms of alzheimer's disease: analysis of the $19 q 13.2$ chromosomal region. Hum Mol Genet. 1994;3:569-74. https://doi.org/ 10.1093/hmg/3.4.569.

33. Mahley RW, Rall SC. Apolipoprotein E: Far more than a lipid transport protein. Annu Rev Genomics Hum Genet. 2000;1:507-37. https://doi. org/10.1146/annurev.genom.1.1.507.

34. Yamazaki Y, Zhao N, Caulfield TR, Liu CC, Bu G. Apolipoprotein E and Alz heimer disease: pathobiology and targeting strategies. Nat Rev Neurol. 2019;15:501-18.

35. Oh U, Gupta R, Krakauer JW, Khandji AG, Chin SS, Elkind MSV. Reversible leukoencephalopathy associated with cerebral amyloid angiopathy. Neurology. 2004;62:494-7. https://doi.org/10.1212/01.WNL.00001 06951.94624.DF.

36. Kinnecom C, Lev MH, Wendell L, Smith EE, Rosand J, Frosch MP, et al. Course of cerebral amyloid angiopathy-related inflammation. Neurology. 2007;68:1411-6.

37. Lim SY, Wesley Thevathasan A, Gonzales M, Mitchell PJ, Evans A. Vasogenic oedema with no mass lesion. J Clin Neurosci. 2008;15:9.

38. Greenberg SM, William Rebeck G, Vonsattel JPG, Gomez-Isla T, Hyman BT. Apolipoprotein E $\epsilon 4$ and cerebral hemorrhage associated with amyloid angiopathy. Ann Neurol. 1995;38:254-9. https://doi.org/10.1002/ ana.410380219.

39. Alonzo NC, Hyman BT, Rebeck GW, Greenberg SM. Progression of cerebral amyloid angiopathy: accumulation of amyloid- $\beta 40$ in affected vessels. J Neuropathol Exp Neurol. 1998;57:353-9. https://doi.org/10. 1097/00005072-199804000-00008.

40. Utter S, Tamboli IY, Walter J, Upadhaya AR, Birkenmeier G, Pietrzik CU, et al. Cerebral small vessel disease-induced apolipoprotein e leakage is associated with alzheimer disease and the accumulation of amyloid $\beta$-protein in perivascular astrocytes. J Neuropathol Exp Neurol. 2008:67:842-56. https://doi.org/10.1097/NEN.0b013e3181836a71.

41. Luo X, Jiaerken Y, Yu X, Huang P, Qiu T, Jia Y, et al. Associations between APOE genotype and cerebral small-vessel disease: a longitudinal study. Oncotarget. 2017:8:44477-89.

42. Bell RD, Winkler EA, Singh I, Sagare AP, Deane R, Wu Z, et al. Apolipoprotein e controls cerebrovascular integrity via cyclophilin A. Nature. 2012;485:512-6. https://doi.org/10.1038/nature11087. 
43. Zhang Z, Chen XY, Baum L, Ng HK, MokV, Wong KS. Association between the apolipoprotein e gene polymorphism and atherosclerotic middle cerebral artery stenosis. Neurologist. 2018;23:47-50.

44. Kosunen O, Talasniemi S, Lehtovirta M, Heinonen O, Helisalmi SM, Mannermaa AM, et al. Relation of coronary atherosclerosis and apolipoprotein e genotypes in alzheimer patients. Stroke. 1995;26:743-8.

45. Thambisetty M, Beason-Held L, An Y, Kraut MA, Resnick SM. APOE $\varepsilon 4$ genotype and longitudinal changes in cerebral blood flow in normal aging. Arch Neurol. 2010;67:93-8.

46. Farrall AJ, Wardlaw JM. Blood-brain barrier: ageing and microvascular disease — systematic review and meta-analysis. Neurobiol Aging. 2009;30:337-52.

47. Montagne A, Nation DA, Sagare AP, Barisano G, Sweeney MD, Chakhoyan A, et al. APOE4 leads to blood-brain barrier dysfunction predicting cognitive decline. Nature. 2020;581:71-6.

48. Salloway S, Gur T, Berzin T, Zipser B, Correia S, Hovanesian V, et al. Effect of APOE genotype on microvascular basement membrane in Alzheimer's disease. J Neurol Sci. 2002;203:183-7.

49. Dickstein DL, Walsh J, Brautigam H, Stockton SD, Gandy S, Hof PR. Role of vascular risk factors and vascular dysfunction in Alzheimer's disease. Mt Sinai J Med. 2010;77:82-102. https://doi.org/10.1002/msj.20155.

50. Johnson ECB, Dammer EB, Duong DM, Ping L, Zhou M, Yin L, et al. Large-scale proteomic analysis of Alzheimer's disease brain and cerebrospinal fluid reveals early changes in energy metabolism associated with microglia and astrocyte activation. Nat Med. 2020;26:769-80.

51. Johnson ECB, Dammer EB, Duong DM, Yin L, Thambisetty M, Troncoso $J C$, et al. Deep proteomic network analysis of Alzheimer's disease brain reveals alterations in RNA binding proteins and RNA splicing associated with disease. Mol Neurodegener. 2018. https://doi.org/10.1186/ s13024-018-0282-4.

52. Bai B, Wang X, Li Y, Chen PC, Yu K, Dey KK, et al. Deep multilayer brain proteomics identifies molecular networks in Alzheimer's disease progression. Neuron. 2020;105:975-991.e7.

53. Zhang J, Keene CD, Pan C, Montine KS, Montine TJ. Proteomics of human neurodegenerative diseases. J Neuropathol Exp Neurol. 2008;67:923-32

54. Manousopoulou A, Gatherer M, Smith C, Nicoll JAR, Woelk CH, Johnson $M$, et al. Systems proteomic analysis reveals that clusterin and tissue inhibitor of metalloproteinases 3 increase in leptomeningeal arteries affected by cerebral amyloid angiopathy. Neuropathol Appl Neurobiol. 2017:43:492-504.

55. Hondius DC, Eigenhuis KN, Morrema THJ, van der Schors RC, van Nierop P, Bugiani M, et al. Proteomics analysis identifies new markers associated with capillary cerebral amyloid angiopathy in Alzheimer's disease. Acta Neuropathol Commun. 2018;6:46. https://doi.org/10.1186/ s40478-018-0540-2.

56. Badhwar A, Brown R, Stanimirovic DB, Haqqani AS, Hamel E. Proteomic differences in brain vessels of Alzheimer's disease mice: normalization by PPARI 3 agonist pioglitazone. J Cereb Blood Flow Metab. 2017;37:1120-36.

57. Pearson A, Ajoy R, Crynen G, Reed JM, Algamal M, Mullan M, et al. Molecular abnormalities in autopsied brain tissue from the inferior horn of the lateral ventricles of nonagenarians and Alzheimer disease patients. BMC Neurol. 2020. https://doi.org/10.1186/ s12883-020-01849-3.

58. Vonsattel JPG, Myers RH, Tessa Hedley-Whyte E, Ropper AH, Bird ED, Richardson EP. Cerebral amyloid angiopathy without and with cerebral hemorrhages: a comparative histological study. Ann Neurol. 1991;30:637-49.

59. Bachmeier C, Shackleton B, Ojo J, Paris D, Mullan M, Crawford F. Apolipoprotein $\mathrm{E}$ isoform-specific effects on lipoprotein receptor processing. NeuroMolecular Med. 2014;16(4):686-96. https://doi.org/10.1007/ s12017-014-8318-6.

60. Ojo J, Eisenbaum M, Shackleton B, Lynch C, Joshi U, Saltiel N, et al. Mural cell dysfunction leads to altered cerebrovascular tau uptake following repetitive head trauma. Neurobiol Dis. 2021;150(105237):S09699961203 05131.

61. Ojo JO, Crynen G, Algamal M, Vallabhaneni P, Leary P, Mouzon B, et al. Unbiased proteomic approach identifies pathobiological profiles in the brains of preclinical models of repetitive mild traumatic brain injury, tauopathy, and amyloidosis. ASN Neuro. 2020;12:1759091420914768. https://doi.org/10.1177/1759091420914768.

62. Vizcaíno JA, Csordas A, Del-Toro N, Dianes JA, Griss J, Lavidas I, et al. 2016 update of the PRIDE database and its related tools. Nucleic Acids Res. 2016;44:D447-56.

63. Krämer A, Green J, Pollard J, Tugendreich S. Causal analysis approaches in ingenuity pathway analysis. Bioinformatics. 2014;30:523-30.

64. Nicoll JAR, Burnett C, Love S, Graham DI, Dewar D, Ironside JW, et al. High frequency of apolipoprotem E $\varepsilon 2$ allele in hemorrhage due to cerebral amyloid angiopathy. Ann Neurol. 1997;41:716-21.

65. McCarron MO, Nicoll JAR. High frequency of apolipoprotein E $\varepsilon 2$ allele is specific for patients with cerebral amyloid angiopathy-related haemorrhage. Neurosci Lett. 1998;247:45-8.

66. Nicoll JAR, McCarron MO. APOE gene polymorphism as a risk factor for cerebral amyloid angiopathy-related hemorrhage. Amyloid. 2001:8:51-5.

67. Schilling S, DeStefano AL, Sachdev PS, Choi SH, Mather KA, DeCarli CD, et al. APOE genotype and MRI markers of cerebrovascular disease. Neurology. 2013;3:292-300.

68. Huang Y, Schwendner SW, Rall SC, Mahley RW. Hypolipidemic and hyperlipidemic phenotypes in transgenic mice expressing human apolipoprotein E2. J Biol Chem. 1996;271:29146-51.

69. Rasmussen KL, Tybjærg-Hansen A, Nordestgaard BG, Frikke-Schmidt R. Plasma levels of apolipoprotein E, APOE genotype, and all-cause and cause-specific mortality in 105949 individuals from a white general population cohort. Eur Heart J. 2019;40:2813-24.

70. Sebastiani P, Raghavachari N. Neuroprotective effect of apoe2: evidence and implication for cognitive aging. Innov Aging. 2019;3:S620.

71. Shinohara M, Kanekiyo T, Tachibana M, Kurti A, Shinohara M, Fu Y, et al. Apoe 2 is associated with longevity independent of alzheimer's disease. Elife. 2020. https://doi.org/10.7554/eLife.62199.

72. Lin AL, Parikh I, Yanckello LM, White RS, Hartz AMS, Taylor CE, et al. APOE genotype-dependent pharmacogenetic responses to rapamycin for preventing Alzheimer's disease. Neurobiol. Dis. 2020.

73. Lin AL, Butterfield DA, Richardson A. mTOR: Alzheimer's disease prevention for APOE4 carriers. Oncotarget. 2016;29:44873.

74. Ohno M. Roles of elF2a kinases in the pathogenesis of Alzheimer's disease. Front Mol Neurosci. 2014;7:22

75. Oliveira MM, Lourenco MV. Integrated stress response: Connecting apoE4 to memory impairment in Alzheimer's disease. J Neurosci. 2016;36:4.

76. Segev Y, Michaelson DM, Rosenblum K. ApoE $\varepsilon 4$ is associated with elF2a phosphorylation and impaired learning in young mice. Neurobiol Aging. 2013;34:863.

77. Dai J, Johnson ECB, Dammer EB, Duong DM, Gearing M, Lah JJ, et al. Effects of APOE genotype on brain proteomic network and cell type changes in Alzheimer's disease. Front Mol Neurosci. 2018. https://doi. org/10.3389/fnmol.2018.00454.

78. Nelson PT, Pious NM, Jicha GA, Wilcock DM, Fardo DW, Estus S, et al. APOE- $\varepsilon 2$ and APOE $\varepsilon 4$ correlate with increased amyloid accumulation in cerebral vasculature. J Neuropathol Exp Neurol. 2013;72:708-15.

79. Rannikmäe K, Kalaria RN, Greenberg SM, Chui HC, Schmitt FA, Samarasekera N, et al. APOE associations with severe CAA-associated vasculopathic changes: collaborative meta-analysis. J Neurol Neurosurg Psychiatry. 2014;85:300-5.

80. MCCarron MO, Nicoll JAR. Apolipoprotein E genotype and cerebral amyloid angiopathy-related hemorrhage. Ann N Y Acad Sci. 2000:903:176-9.

81. Hawkes CA, Sullivan PM, Hands S, Weller RO, Nicoll JAR, Carare RO. Disruption of arterial perivascular drainage of amyloid- $\beta$ from the brains of mice expressing the human APOE \&4 allele. PLoS One. 2012;7:e41636.

82. Thal DR, Griffin WST, de Vos RAI, Ghebremedhin E. Cerebral amyloid angiopathy and its relationship to Alzheimer's disease. Acta Neuropathol. 2008;6:599-609.

83. Deane R, Sagare A, Hamm K, Parisi M, Lane S, Finn MB, et al. apoE isoform-specific disruption of amyloid $\beta$ peptide clearance from mouse brain. J Clin Invest. 2008;118:4002-13.

84. Thal DR, Larionov S, Abramowski D, Wiederhold KH, Van Dooren $\mathrm{T}$, Yamaguchi $\mathrm{H}$, et al. Occurrence and co-localization of amyloid $\beta$-protein and apolipoprotein E in perivascular drainage channels of wild-type and APP-transgenic mice. Neurobiol Aging. 2007;28:1221-30. 
85. Rolyan H, Feike AC, Upadhaya AR, Waha A, Van Dooren T, Haass C, et al. Amyloid- $\beta$ protein modulates the perivascular clearance of neuronal apolipoprotein e in mouse models of Alzheimer's disease. J Neural Transm. 2011;118:699-712.

86. Zekonyte J, Sakai K, Nicoll JAR, Weller RO, Carare RO. Quantification of molecular interactions between $A p o E$, amyloid-beta $(A \beta)$ and laminin: relevance to accumulation of $A B$ in Alzheimer's disease. Biochim Biophys Acta Mol Basis Dis. 2016;1862:1047-53.

87. Yamaguchi H, Ishiguro K, Sugihara S, Nakazato Y, Kawarabayashi T, Sun X, et al. Presence of apolipoprotein E on extracellular neurofibrillary tangles and on meningeal blood vessels precedes the Alzheimer $\beta$-amyloid deposition. Acta Neuropathol. 1994;88:413-9.

88. Fullerton SM, Shirman GA, Strittmatter WJ, Matthew WD. Impairment of the blood-nerve and blood-brain barriers in apolipoprotein E knockout mice. Exp Neurol. 2001;169:13-22.

89. Donahue JE, Johanson CE. Apolipoprotein E, amyloid- $\beta$, and bloodbrain barrier permeability in Alzheimer disease. J Neuropathol Exp Neurol. 2008;67:261-70. https://doi.org/10.1097/NEN.0b013e31816a0dc8.

90. Kim J, Basak JM, Holtzman DM. The role of apolipoprotein E in Alzheimer's Disease. Neuron. 2009;63:287-303.

91. Shinohara M, Petersen RC, Dickson DW, Bu G. Brain regional correlation of amyloid- $\beta$ with synapses and apolipoprotein $E$ in non-demented individuals: potential mechanisms underlying regional vulnerability to amyloid- $\beta$ accumulation. Acta Neuropathol. 2013;125:535-47. https:// doi.org/10.1007/s00401-013-1086-9.

92. Pirttilä T, Soininen H, Heinonen O, Lehtimäki T, Bogdanovic N, Paljärvi $\mathrm{L}$, et al. Apolipoprotein $\mathrm{E}$ (apoE) levels in brains from Alzheimer disease patients and controls. Brain Res. 1996;722:71-7.

93. Lambert JC, Pérez-Tur J, Dupire MJ, Galasko D, Mann D, Amouyel P, et al. Distortion of allelic expression of apolipoprotein E in Alzheimer's disease. Hum Mol Genet. 1997;6:2151-4.

94. Harr SD, Uint L, Hollister R, Hyman BT, Mendez AJ. Brain expression of apolipoproteins $E_{1} J$, and A-I in Alzheimer's disease. J Neurochem. 1996;66:2429-35.

95. Growdon JH, Locascio JJ, Corkin S, Gomez-Isla T, Hyman BT. Apolipoprotein $\mathrm{E}$ genotype does not influence rates of cognitive decline in Alzheimer's disease. Neurology. 1996;47:444-8.

96. Bray NJ, Jehu L, Moskvina V, Buxbaum JD, Dracheva S, Haroutunian V, et al. Allelic expression of APOE in human brain: effects of epsilon status and promoter haplotypes. Hum Mol Genet. 2004;13:2885-92.

97. Bertrand P, Poirier J, Oda T, Finch CE, Pasinetti GM. Association of apolipoprotein E genotype with brain levels of apolipoprotein $E$ and apolipoprotein J (clusterin) in Alzheimer disease. Brain Res Mol Brain Res. 1995;33:174-8.

98. Beffert U, Cohn JS, Petit-Turcotte C, Tremblay M, Aumont N, Ramassamy $C$, et al. Apolipoprotein $E$ and $\beta$-amyloid levels in the hippocampus and frontal cortex of Alzheimer's disease subjects are disease-related and apolipoprotein E genotype dependent. Brain Res. 1999;843:87-94.

99. Halliday MR, Rege SV, Ma Q, Zhao Z, Miller CA, Winkler EA, et al. Accelerated pericyte degeneration and blood-brain barrier breakdown in apolipoprotein E4 carriers with Alzheimer's disease. J Cereb Blood Flow Metab. 2016;36:216-27. https://doi.org/10.1038/jcbfm.2015.44.
100. Nation DA, Sweeney MD, Montagne A, Sagare AP, D'Orazio LM, Pachicano $\mathrm{M}$, et al. Blood-brain barrier breakdown is an early biomarker of human cognitive dysfunction. Nat Med. 2019;25:270-6.

101. Nortley R, Korte N, Izquierdo P, Hirunpattarasilp C, Mishra A, Jaunmuktane $Z$, et al. Amyloid b oligomers constrict human capillaries in Alzheimer's disease via signaling to pericytes. Science. 2019;365:6450.

102. Khennouf L, Gesslein B, Brazhe A, Octeau JC, Kutuzov N, Khakh BS, et al. Active role of capillary pericytes during stimulation-induced activity and spreading depolarization. Brain. 2018;141:2032-46.

103. Hamilton NB. Pericyte-mediated regulation of capillary diameter: a component of neurovascular coupling in health and disease. Front Neuroenergetics. 2010. https://doi.org/10.3389/fnene.2010.00005/abstr act.

104. Shi Y, Manis M, Long J, Wang K, Sullivan PM, Serrano JR, et al. Microglia drive APOE-dependent neurodegeneration in a tauopathy mouse model. J Exp Med. 2019;216:2546-61.

105. Rodriguez GA, Tai LM, LaDu MJ, Rebeck GW. Human APOE4 increases microglia reactivity at $A \beta$ plaques in a mouse model of $A \beta$ deposition. J Neuroinflammation. 2014;11:111.

106. Vitek MP, Brown CM, Colton CA. APOE genotype-specific differences in the innate immune response. Neurobiol Aging. 2009;30:1350-60.

107. Zhao L, Lin S, Bales KR, Gelfanova V, Koger D, DeLong C, et al. Macrophage-mediated degradation of $\beta$-amyloid via an apolipoprotein e isoform-dependent mechanism. J Neurosci. 2009;29:3603-12.

108. Lin YT, Seo J, Gao F, Feldman HM, Wen HL, Penney J, et al. APOE4 causes widespread molecular and cellular alterations associated with Alzheimer's disease phenotypes in human iPSC-derived brain cell types. Neuron. 2018;98:1141-1154.e7.

109. Shi Y, Yamada K, Liddelow SA, Smith ST, Zhao L, Luo W, et al. ApoE4 markedly exacerbates tau-mediated neurodegeneration in a mouse model of tauopathy. Nature. 2017;549:523-7. https://doi.org/10.1038/ nature24016.

110. Krasemann S, Madore C, Cialic R, Baufeld C, Calcagno N, El Fatimy R, et al. The TREM2-APOE pathway drives the transcriptional phenotype of dysfunctional microglia in neurodegenerative diseases. Immunity. 2017:47:566-581.e9.

111. Shi Y, Yamada K, Liddelow SA, Smith ST, Zhao L, Luo W, et al. ApoE4 markedly exacerbates tau-mediated neurodegeneration in a mouse model of tauopathy, Alzheimer's Disease Neuroimaging Initiative. Nature. 2017:549:523.

112. Liu T, Zhu B, Liu Y, Zhang X, Yin J, Li X, et al. Multi-omic comparison of Alzheimer's variants in human ESC-derived microglia reveals convergence at APOE. J Exp Med. 2020. https://doi.org/10.1084/jem.20200474.

113. Cruz Hernández JC, Bracko O, Kersbergen CJ, Muse V, Haft-Javaherian M, Berg $\mathrm{M}$, et al. Neutrophil adhesion in brain capillaries reduces cortical blood flow and impairs memory function in Alzheimer's disease mouse models. Nat Neurosci. 2019;22:413-20.

\section{Publisher's Note}

Springer Nature remains neutral with regard to jurisdictional claims in published maps and institutional affiliations.

\footnotetext{
Ready to submit your research? Choose BMC and benefit from:

- fast, convenient online submission

- thorough peer review by experienced researchers in your field

- rapid publication on acceptance

- support for research data, including large and complex data types

- gold Open Access which fosters wider collaboration and increased citations

- maximum visibility for your research: over $100 \mathrm{M}$ website views per year
}

At BMC, research is always in progress.

Learn more biomedcentral.com/submissions 